\title{
Polymorphic Type Inference for Scripting Languages with Object Extensions
}

\author{
Tian Zhao \\ University of Wisconsin - Milwaukee, \\ Milwaukee, Wisconsin, USA \\ tzhao@uwm.edu
}

\begin{abstract}
This paper presents a polymorphic type inference algorithm for a small subset of JavaScript. The goal is to prevent accessing undefined members of objects. We define a type system that allows explicit extension of objects through add operation and implicit extension through method calls. The type system also permits strong updates and unrestricted extensions to new objects. The type inference algorithm is modular so that each function definition is only analyzed once and larger programs can be checked incrementally.
\end{abstract}

Categories and Subject Descriptors F.3.2 [Logics and Meanings of Programs]: Semantics of Programming Languages - program analysis; F.3.3 [Logics and Meanings of Programs]: Studies of Program Constructs - type structure

General Terms Languages, Theory, Verification

Keywords Type inference, static types, JavaScript, dynamic languages

\section{Introduction}

JavaScript is an object-based scripting languages widely used for Web applications. As a dynamic language, JavaScript allows objects be modified at runtime for updating existing members, adding new members, or deleting members. Functions can be added to objects to become methods. Object members can be replaced by values of different types. This dynamic nature can result in runtime errors such as accessing an undefined object member or invoking an object property that is not a function. Since JavaScript is dynamically typed, there is no static approach to automatically determine the type information of objects at each program point and runtime type tests are often used to examine the properties of objects.

In JavaScript, objects may be created directly as object literals or by invoking constructor functions through new operator. After an object is created, it can be extended with new members. The extension may be explicit through assignment. For example the statements below extend empty object $\mathrm{x}$ with a new member of number type.

$\operatorname{var} \mathrm{x}=\{\}$

$\mathrm{x} \cdot \mathrm{m}=1$;
If we write the initial type of $\mathrm{x}$ as [ ], then its type at line 2 becomes [m : num], where [] represents the type of empty object and num is the type of numbers. The extension may also be implicit through function calls.

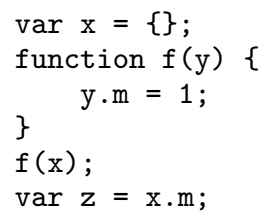

After the call to $f$, the type of $x$ also has member $m$.

Because of this kind of extensions, an object may have different sets of members during execution and a variable pointing to the object should be assigned object types to reflect the correct set of members at each program point. A type inference algorithm has to statically track the members added to the object variable at different parts of a program.

To address this problem, type inference algorithms [4, 12, 25] were developed to statically track the members of objects. However, these type systems are rather restrictive in that they infer monomorphic types and whole program analysis is needed for each program. These type systems may also have difficulty in dealing with cases such as polymorphic functions and container objects. A more useful type inference algorithm needs to support both object extensions and polymorphism.

Polymorphism promotes code reuse and is an essential part of object-oriented programming. JavaScript functions exhibit parametric polymorphism when invoked with different types of arguments. The arguments to a JavaScript function include an implicit self pointer accessible via this variable. A function may be assigned as methods to different types of objects and if the function is invoked as a method, then the self pointer of the function is bound to the receiver object of the call. It is too restrictive to assign monomorphic type to a function since different arguments to the function are forced to be have common super types.

For example, the constructor function $\mathrm{F}$ shown below can be used to create different types of objects.

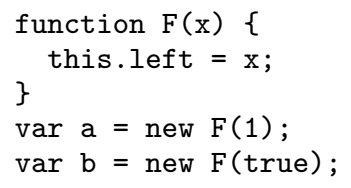

The type for $\mathrm{F}$ can be $t \rightarrow[$ left $: t]$ so that the types of $\mathrm{a}$ and $\mathrm{b}$ are [left : num] and [left : bool] respectively.

However, subtyping can complicate type inference. The function copy shown below requires an object with member left and return the object after adding or updating a member right. 


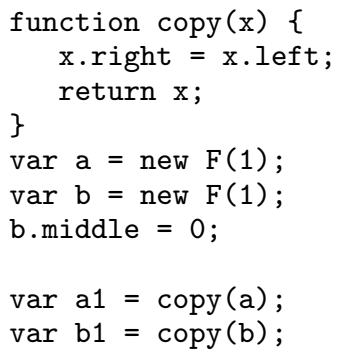

It is not immediately clear how to assign a type to copy to support polymorphism. By Anderson et al.'s design [4], we can assign a type $\tau \rightarrow \tau^{\prime}$ to the function copy, where

$$
\begin{aligned}
\tau & =[\text { left }:(\text { num }, \bullet), \text { right }:(\text { num }, \circ)] \\
\tau^{\prime} & =[\text { left }:(\text { num }, \bullet), \text { right }:(\text { num }, \bullet)]
\end{aligned}
$$

and $\bullet$ labels definitely present members while $\circ$ labels potential members of objects. The types of $a$ and $b$ must have potential member right as well since they are subtypes of the parameter type of copy. There are two problems with this approach. One is that the return type of the constructor $\mathrm{F}$ is forced to have a potential member right of num type, which is unnecessarily restrictive. Another problem is that the statement

$$
\text { var } c=b 1 . \text { middle; }
$$

would not be typable even though it is safe. Recency type system [12] may also have similar problem with this example as it needs to assign a summary object type (that have a fixed set of members) to the parameter of copy since it is not a precise object.

To address the above problems, we define a polymorphic, constraint-based type inference algorithm for a small subset of JavaScript with support for dynamic extension and update of object members.

We can avoid the first problem by assigning a kind of extensive object types called pro-type to new objects (e.g. a and $\mathrm{b}$ in previous example) so that their types can be extended with new members when necessary but not before that (e.g. by function call copy (a) and copy (b)). This design is similar to the singleton object type in recency type system though pro-types are only for local variables.

Specifically, we use two kinds of object types: pro-type and obj-type. The pro-types are assigned to local variables that refer to objects created in the local scope and we allow strong updates (where members of an object can be replaced by values of different types) and unrestricted extensions to the variables of pro-types. The obj-types are assigned to other local variables, function parameters, and object fields. A pro-type is uniquely associated with an object while an obj-type may be related to multiple objects. A variable of obj-type can also be extended though it may not have strong updates. Our type system also keeps track of members added to an object by implicit extension through function parameters and self pointer. The latter is called self-inflicted extension [7] - the extension that an object made to itself upon receiving a message.

For the second problem, our algorithm uses a generalized form of types adopted from the recursively constrained (rc) types [8], which was used for polymorphic type inference for an objectoriented language. The rc types have the form of $\tau \backslash C$, where $C$ is a set of type constraints, each of the form $\tau_{1} \leq \tau_{2}$. The rc type is a generalization of recursive types and it can model a restricted form of union and intersection type with multiple upper bounds and lower bounds on the same type variable. The type that we infer for the function copy is parameterized so that each application of copy results in a new version of the type. As a result, the call copy (b) returns an object with a member middle while copy (a) does not.

Our system has its limitations. We do not consider JavaScript features such as accessing objects as associative arrays, function prototypes, variadic functions, and eval function. Also, we do not allow strong update of object types through function or method calls. For example, the last line in the code fragment below is not typable even though it will run correctly.

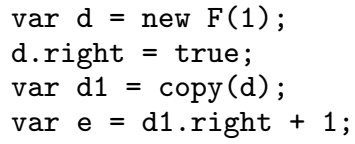

That is, we cannot overwrite the boolean type of the right member of $d$ when it is assigned the integer value of its left member in function copy. In fact, the type of the d1.right is inferred as the super type of both num and bool so that d1.right +1 is not typable. We will consider these limitations in future work.

In summary, we present a type inference algorithm for a small subset of JavaScript language that

1. infers polymorphic function types,

2. keeps track of object extensions through direct assignments and function/method calls, and

3. allows strong updates and flexible extensions to new objects in local scope.

In the rest of paper, we first present some motivating examples in Section 2 and then we give an informal discussion of our approach in Section 3. Next, we formalize a type system on a language that models some core features of JavaScript. We present the syntax and typing rules in Section 4. We explain the type inference algorithm and the simplification of the inferred types in Section 5 . The operational semantics and soundness proof of the type system are in the appendix.

\section{Motivating examples}

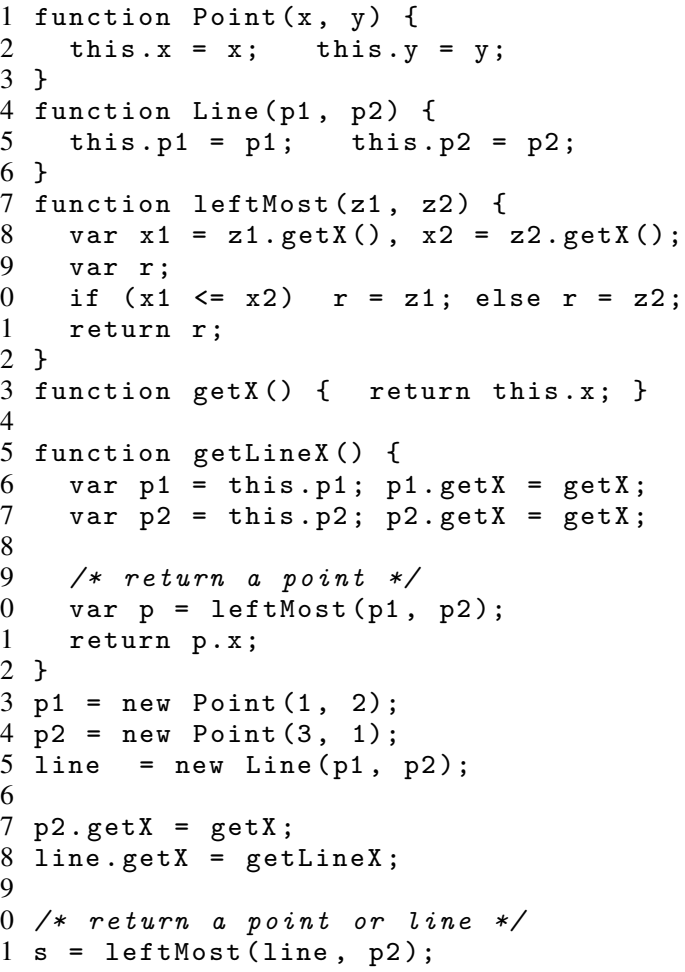

Figure 1. Object extension and polymorphism 
Figure 1 is an example of object extension and polymorphism, where Point and Line are constructor functions that return new point and line objects respectively. The function leftMost takes two geometry objects (either line or point) and return the one on the left based on the left-most $\mathrm{x}$-coordinate of the geometry. The function assumes that the arguments have a getX method that returns the left-most $\mathrm{x}$-coordinate. Before calling the leftMost function with a point or line object as argument, it is necessary to add appropriate getX methods to the geometries. For example, before the call leftMost (line, p2) on line 31, function getX is added to the point object p2 (line 27) and getLineX is added to the line object (line 28).

Notice that the call leftMost(line, p2) returns either a point or a line object. However, in function getLineX , the function call leftMost (p1, p2) (line 20) returns a point object. The statement at line 21 assumes the returned object is a point and read its member $\mathrm{x}$. This polymorphic use of function leftMost makes it difficult to assign static types to the parameter and return types. For example, we cannot assign a Point type or Line type to the parameters of leftMost since it accepts both kinds of argument. It is possible that the function may take other kinds of argument such as polygon. Parametric function type does not work in this case either. If we assign variable types $\alpha_{1}$ and $\alpha_{2}$ to the function parameter $\mathrm{z} 1$ and $z 2$ respectively, then the return type is neither $\alpha_{1}$ nor $\alpha_{2}$ but an upper bound of the union type $\alpha_{1} \vee \alpha_{2}$.

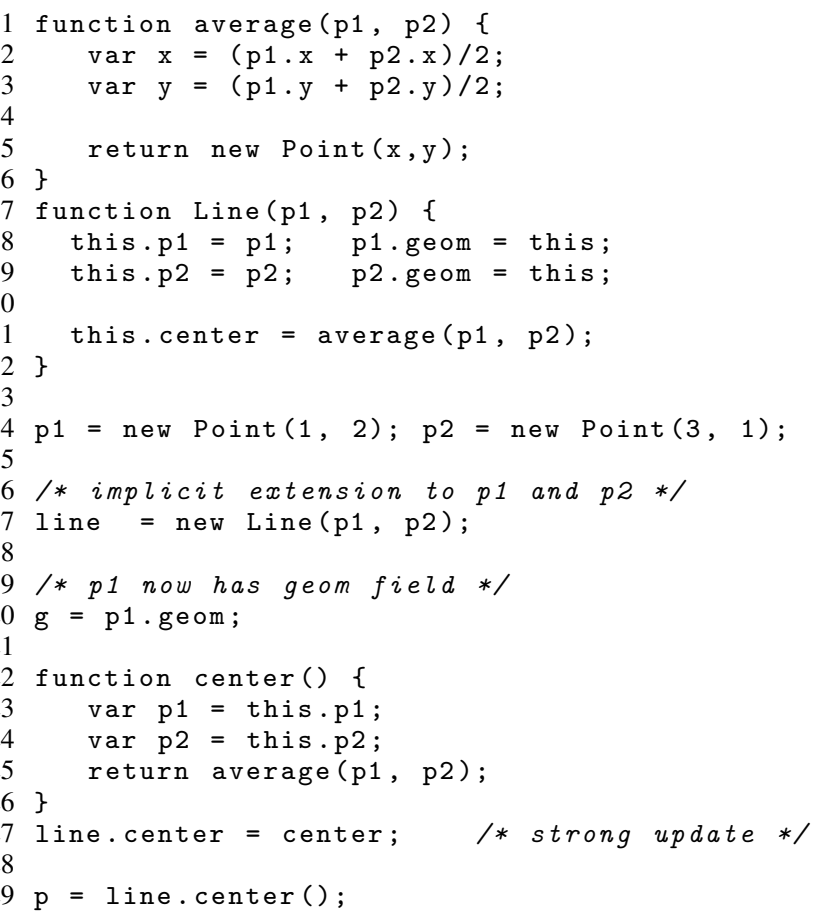

Figure 2. Implicit extension and strong update

JavaScript objects may be extended after being passed as arguments to functions or as receivers of method calls. Figure 2 shows such an example, where point objects passed to the Line constructor are extended with a field geom that points to the line object (line 8 and 9). After call to the Line constructor, the point $\mathrm{p} 1$ has a field geom and its access is allowed (line 20).

Figure 2 also includes an example of strong update. The Line constructor adds a field center to each new line object, which is the center point of the line. If a line's end points may change, it may be better to have a method to compute the center point based on the current end points. To do this, we simply replace the center member of the line object with a function center at line 27. This is strong update since the new member is a function while the existing member is an object.

\section{Approach}

In this section, we give an informal description of our type system using the motivating examples.

\subsection{Function types}

In our design, we assume that a program consists of a sequence of function declarations (including constructor functions) and a main program (consists of a sequence of statements). For each function of the form

we infer a rc type

$$
\text { function } f(x)\{\langle\text { function body }\rangle\} \text {, }
$$

$$
\tau \backslash C
$$

where $\tau$ is the type of the function and $C$ is a set of constraints that constrain $\tau$ and other types related to $\tau$.

The set $C$ is formed through the type derivation of the statements of the function body. After each statement is analyzed, $C$ may be added with more constraints. The set $C$ must be consistent after each statement is analyzed so that its closure does not contain inconsistent constraints such as num $\leq$ bool. We do not directly verify subtyping relation between types. Instead, to check whether $\tau_{1} \leq \tau_{2}$, we add this constraint to the current constraint set $C$ and check if $C \cup\left\{\tau_{1} \leq \tau_{2}\right\}$ is consistent. One problem with this approach is that a large number of constraints may be generated for a function while many of them are not needed after the function is checked. To reduce the size of constraint set, we define simplification rules to remove redundant constraints from $C$ and only keep those constraints related to the parameter types and return types of the functions.

For the rest of the section, we will refer to $C$ as the constraint set of the current context.

To support parametric polymorphism, for each reference of a function/constructor of the type $\tau_{f} \backslash C_{f}$, we instantiate the type by replacing the free variables in $\tau_{f}$ and $C_{f}$ with fresh variables using a renaming function $\Psi$ and add $\Psi C_{f}$ to $C$.

\subsection{Object types}

We have two kinds of object types: pro-type and obj-type. A protype (denoted by meta variable $\varsigma$ ) is uniquely associated with an object such as the self object of a constructor function or a newly instantiated object.

All pro-types are initially type variables. We use meta variable $\beta$ for the initial type of the self object of a constructor function and use meta variable $\gamma$ for the initial type of a new object. If a variable $y$ of type $\varsigma$ is explicitly extended through assignment such as $y . m=y^{\prime}$, we give $y$ a new pro-type

$$
\varsigma \leftarrow(m, t),
$$

where the constraint $\tau \leq t$ is added to $C$ and $\tau$ is the type of $y^{\prime}$. The type $\varsigma \leftarrow(m, t)$ has the meaning that $\varsigma$ is added a member $m$ of type $t$ and if this member already exists in $\varsigma$, then it is replaced. This representation permits strong update.

In the Point function in Figure 1, the initial type of this variable is a variable pro-type $\beta_{p}$ that represents empty object. After line 2, the type of this becomes

$$
\varsigma_{p}=\left(\beta_{p} \leftarrow\left(x, t_{1}\right)\right) \leftarrow\left(y, t_{2}\right),
$$

with the constraint set $\left\{t_{x} \leq t_{1}, t_{y} \leq t_{2}\right\}$, where $t_{x}, t_{y}$ are parameter types of the constructor. The rc type of Point is then

$$
t_{x} \times t_{y} \rightarrow \varsigma_{p} \backslash\left\{t_{x} \leq t_{1}, t_{y} \leq t_{2}\right\} .
$$


We let the type constructor $\leftarrow$ be left associative so that we can write $\left(\beta_{p} \leftarrow\left(x, t_{1}\right)\right) \leftarrow\left(y, t_{2}\right)$ as $\beta_{p} \leftarrow\left(x, t_{1}\right) \leftarrow\left(y, t_{2}\right)$.

If we instantiate a point object as below

$\operatorname{var} \mathrm{p}=$ new Point $(1,2)$;

then the type of $\mathrm{p}$ is $\gamma_{p}$ with the constraints

$$
\begin{aligned}
&\left\{\beta_{p}^{\prime} \leftarrow\left(\mathrm{x}, t_{1}^{\prime}\right)\right. \leftarrow\left(\mathrm{y}, t_{2}^{\prime}\right) \leq \gamma_{p}, \\
&\text { num } \left.\leq t_{x}^{\prime}, t_{x}^{\prime} \leq t_{1}^{\prime}, \text { num } \leq t_{y}^{\prime}, t_{y}^{\prime} \leq t_{2}^{\prime}\right\} .
\end{aligned}
$$

The type $\gamma_{p}$ is a pro-type variable representing the initial type of the new object $p$ and the primed type variables are instances of the free variables in the rc type of Point.

An obj-type may be related to multiple objects and is also initially a type variable (denoted by meta variable $t$ ). We give obj-types to function parameters, object members, and the local variables that are not uniquely associated with an object. An object of obj-type may be extended and we write the type of the extended object as $(t, M)$, where $M$ is a meta variable representing the names of members added to $t$. If a variable $y$ of an obj-type $t$ is explicitly extended in $y \cdot m=y^{\prime}$, then the type of $y$ becomes $(t,\{m\})$, which means that $m$ is a member added to $t$ but the type of $m$ is not changed. Also, the constraints

$$
\left\{t \leq\left[m^{-}: t^{\prime}\right], \tau \leq t^{\prime}\right\}
$$

are added to $C$, where $\tau$ is the type of $y^{\prime}$ and constraint of the form $t \leq\left[m^{-}:{ }_{-}\right]$is added for each write on obj-types.

\subsection{Reading an object member}

In general, we add constraints of the form

$$
\left\{\tau \leq\left[m^{+}: t\right], m \in \tau\right\},
$$

for each read of a member $m$ (including method call) on an object of the type $\tau$, where $m \in \tau$ is a special type of constraints that check whether $\tau$ or its type lower bounds (if $\tau$ is a type variable) have the member $m$. A constraint of the form $m \in \beta$ is immediately inconsistent since $\beta$ represents an empty object.

For example, the rc type of the function getX in Figure 1 is

$$
t \rightarrow t_{g} \backslash\left\{t \leq\left[\mathrm{x}^{+}: t_{g}\right], \mathrm{x} \in t\right\},
$$

where $t$ is the type of this variable in getX. The constraints $\left\{t \leq\left[\mathrm{x}^{+}: t_{g}\right], \mathrm{x} \in t\right\}$ correspond to reading the member $\mathrm{x}$ on this variable. Since $t$ is a type variable, its lower bounds must have the member $\mathrm{x}$. Within the rc type of getX, $t$ has no lower bound but when getX is invoked on an object, the lower bounds of $t$ must have a member $\mathrm{x}$.

Consider the code fragment

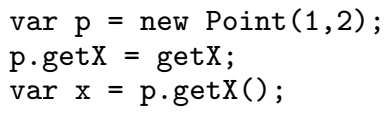

for p.getX $=$ getX, our type system instantiates the type of getX to $t^{\prime} \rightarrow t_{g}^{\prime}$ and add $\left\{t^{\prime} \leq\left[\mathrm{x}^{+}: t_{g}^{\prime}\right], \mathrm{x} \in t^{\prime}\right\}$ to $C$. For $\mathrm{p}$. $\operatorname{getX}()$, the type system adds some constraints to $C$ so that its closure includes a constraint $\gamma_{p} \leq t^{\prime}$, where $\gamma_{p}$ is the type of $\mathrm{p}$ as defined previously. By transitivity, $\beta^{\prime} \leftarrow\left(\mathrm{x}, t_{1}^{\prime}\right) \leftarrow\left(\mathrm{y}, t_{2}^{\prime}\right)$ is a lower bound of $t^{\prime}$ and it has the member $\mathrm{x}$.

\subsection{Polymorphism}

To illustrate parametric polymorphism, consider the leftMost function invoked on line 20 and line 31 of Figure 1. The type of the function can be written as

$$
\begin{aligned}
& \left\{t_{1} \leq\left[\operatorname{getX} X^{+}: t_{3}\right], t_{2} \leq\left[\operatorname{get} X^{+}: t_{4}\right],\right. \\
& \text { getX } \in t_{1}, \operatorname{getX} \in t_{2}, \\
\kappa=t_{1} \times t_{2} \rightarrow t \backslash \quad & t_{3} \leq t_{5} \rightarrow t_{7}, t_{4} \leq t_{6} \rightarrow t_{8}, \\
& t_{1} \leq t_{5}, t_{2} \leq t_{6}, t_{1} \leq t, t_{2} \leq t \\
& \left.t_{7} \leq \text { num }, t_{8} \leq \text { num }\right\} .
\end{aligned}
$$

Each time leftMost is called, we instantiate $\kappa$ by renaming its free variables $t$ and $t_{1}-t_{8}$ to some fresh variables.

To assign types for the statement var $\mathrm{p}=\operatorname{leftMost}(\mathrm{p} 1, \mathrm{p} 2)$ at line 20 (within the function getLineX), we instantiate $\kappa$ to

$$
t_{1}^{\prime} \times t_{2}^{\prime} \rightarrow t^{\prime} \backslash C^{\prime} \text {, where }\left\{t_{1}^{\prime} \leq t^{\prime}, t_{2}^{\prime} \leq t^{\prime}\right\} \subseteq C^{\prime} .
$$

Assume the types of $\mathrm{p} 1$ and $\mathrm{p} 2$ are $\tau_{1}$ and $\tau_{2}$ respectively. Then

$$
C^{\prime} \cup\left\{\tau_{1} \leq t_{1}^{\prime}, \tau_{2} \leq t_{2}^{\prime}\right\} \subseteq C .
$$

At line 21, the statement $\mathrm{p} . \mathrm{x}$ adds a constraint $\mathrm{x} \in t^{\prime}$ and since $\tau_{1}$ and $\tau_{2}$ are lower bounds of $t^{\prime}$, they need to have the member $\mathrm{x}$. This is satisfied since getLineX is used as a method of a line object so that $\mathrm{p} 1$ and $\mathrm{p} 2$ are end points of the line.

To assign types for the statement $\mathrm{s}=$ leftMost (line, $\mathrm{p} 2$ ) at line 31 , we instantiate $\kappa$ to

$$
t_{1}^{\prime \prime} \times t_{2}^{\prime \prime} \rightarrow t^{\prime \prime} \backslash C^{\prime \prime} \text {, where }\left\{t_{1}^{\prime \prime} \leq t^{\prime \prime}, t_{2}^{\prime \prime} \leq t^{\prime \prime}\right\} \subseteq C^{\prime \prime} .
$$

The types of line and $\mathrm{p} 2$ are the lower bounds of $t^{\prime \prime}$, which is the type of the variable $s$. Consequently, we cannot treat $s$ as a point or line. We can only access members in s that are defined in both objects such as getX.

\subsection{Implicit extension}

Objects may be extended implicitly when they are passed as arguments to function calls. As an example, consider the constructor Line in Figure 2 that extends its two parameters with a field geom that points to the line object being constructed. After a line object is instantiated (line 17), the arguments $\mathrm{p} 1$ and $\mathrm{p} 2$ should have a new field geom. To model this behavior, we write the type of Line as

$$
\begin{aligned}
\left\{t_{1}\right. & \left.\leq \text { geom }^{-}: t_{\mathrm{g} 1}\right], \\
t_{2} & \left.\leq \text { geom }^{-}: t_{\mathrm{g} 2}\right] \\
t_{1} & \leq t_{\mathrm{p} 1}, t_{2} \leq t_{\mathrm{p} 2} \\
\varsigma_{1} & \left.\leq t_{\mathrm{g} 1}, \varsigma_{2} \leq t_{\mathrm{g} 2}, \ldots\right\}
\end{aligned}
$$

where

$$
\begin{aligned}
\varsigma_{1} & =\beta \leftarrow\left(\mathrm{p} 1, t_{\mathrm{p} 1}\right), \\
\varsigma_{2} & =\varsigma_{1} \leftarrow\left(\mathrm{p} 2, t_{\mathrm{p} 2}\right), \\
\varsigma & =\varsigma_{2} \leftarrow(\text { center }, t) .
\end{aligned}
$$

For clarity, the constraints related to the addition of the member center on line 11 are omitted.

In general, a function type is written as

$$
(t, M) \times\left(t^{\prime}, M^{\prime}\right) \rightarrow \tau \backslash C
$$

where $M$ and $M^{\prime}$ represent the set of members extended through the parameters. In this example, the extended member is geom. At line 17, if the type of $\mathrm{p} 1$ is $\tau_{1}$ before the call, then its type becomes $\left(\tau_{1},\{\right.$ geom $\left.\}\right)$ after the call and accessing this member on $\mathrm{p} 1$ is allowed (line 20).

\subsection{Strong update}

A variable of pro-type can receive strong update to replace an existing member with one of different type. In Figure 2, the assignment line. center $=$ center $($ line 27) replaces the existing center (a point object) of a line object with a function. If the line object at line 27 has a type $\varsigma_{l}$, then after the assignment, the type becomes

$$
\left.\varsigma_{l} \leftarrow \text { (center, } t\right) \text {, where } \tau_{c} \leq t \in C,
$$


and $\tau_{c}$ is an instance of the type of the function center.

\section{Formalization}

In this section, we present a formalization of our type system. We first explain the syntax and the typing rules. The type inference algorithm is given in Section 5. The operational semantics and type soundness proof are in the appendix.

\subsection{Syntax}

We select a small subset of the JavaScript language that includes member select, member update/add, method calls, object creation, and branch statement with syntax shown in Figure 3. We distinguish constructor function and regular function with the naming convention that constructor function name starts with an upper case letter. We do not include function calls since its behavior is similar to that of method calls when the receiver object is empty. In fact, regular function calls in JavaScript will substitute this pointer of the called function with the global object [6].

$$
\begin{aligned}
& P \quad::=F n_{i}{ }^{i \in 1 . . n} s \\
& \begin{aligned}
F n::= & \text { function } f(x)\{s ; \text { return } y\} \\
& \mid \text { function } F(x)\{s\}
\end{aligned} \\
& s \quad::= \\
& x=z \\
& x=\text { new } F\left(y^{\prime}\right) \\
& x=y . m \\
& x=y \cdot m\left(y^{\prime}\right) \\
& y \cdot m=y^{\prime} \\
& s ; s^{\prime} \\
& \text { if }(x)\{s\} \text { else }\left\{s^{\prime}\right\}
\end{aligned}
$$

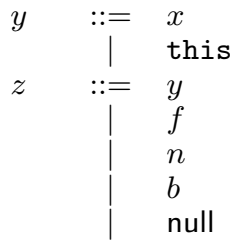

Program

function

constructor

statements

assignment

new object

member select

method call

member update/add

sequence

if statement

local variables self reference

function identifier number

boolean

null value

\section{Figure 3. Syntax}

The syntax of a function body consists of a sequence of statements and a return statement. For simplicity, we write object creation. member select, and method call in the form of assignments and each expression is assigned to a variable so that there is no nested expressions in the statements. We omit the var declaration of variables with the understanding that variables appearing within a function are locally scoped. The body of a constructor function has a sequence of statements but no return statement since each time a constructor function is called through new operator, this pointer of the function is given a new empty object and after the body is executed, this object is returned.

The meta variable $f$ ranges over the names of regular functions, $F$ ranges over the names of constructor functions, and $m$ ranges over member names. A program $P$ consists of a one or more function/constructor definitions and a main statement $s$.

\subsection{Types}

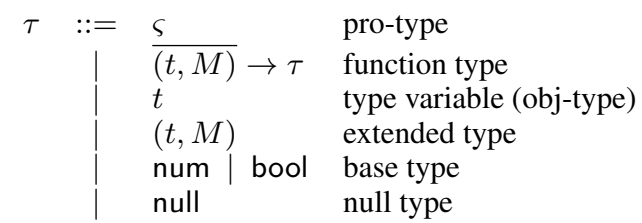

The meta variable $\tau$ ranges over type variables, function types, extended types, pro-types, primitive types (num and bool), and null type. A type variable $t$ may be given to a variable that references any kind of values and when the value is an object, then $t$ is considered an obj-type. An extended type $(t, M)$ is assigned to an obj-type variable after it is extended. In a function type

$$
\overline{(t, M)} \rightarrow \tau,
$$

we write $\overline{(t, M)}$ to represent the types of one or more parameters and it is either $\left(t_{1}, M_{1}\right) \times\left(t_{2}, M_{2}\right)$ for a function (where $t_{1}$ is the type of the self pointer) or $(t, M)$ for a constructor. In the previous examples, we omit the member set $M$ in the parameter types whenever it is empty.

$$
\begin{aligned}
& \varsigma::=\beta \quad \text { empty pro-type } \\
& \mid \begin{array}{ll}
\gamma & \text { initial instance pro-type } \\
(\varsigma, M) & \text { implicitly extended pro-type } \\
\varsigma \leftarrow(m, t) & \text { explicitly extended pro-type } \\
\left(\varsigma, \varsigma^{\prime}\right) & \text { merged pro-type }
\end{array}
\end{aligned}
$$

The pro-types are given to variables that reference new objects. The self pointer of a constructor is initially given variable type $\beta$ that represents empty object. A pro-type $\varsigma$ maybe extended directly by assignment to $\varsigma \leftarrow(m, t)$ or indirectly through function/method calls to $(\varsigma, M)$. If a variable has different pro-types $\varsigma$ and $\varsigma^{\prime}$ in the two branches of an if statement, then we merge the types into $\left(\varsigma, \varsigma^{\prime}\right)$. When a new object is instantiated, the variable that holds the new object is given a pro-type $\gamma$. The closure of the current constraint set will include a constraint of the form $\varsigma \leq \gamma$, where $\varsigma$ is the return type of the constructor. We distinguish this type from the empty pro-type $\beta$ since a constraint of the form $\mathrm{m} \in \beta$ is immediately inconsistent (which indicates that the member $\mathrm{m}$ is not defined) while $\mathrm{m} \in \gamma$ is not. For example, given the statement $\mathrm{p} 1=$ new Point $(1,2)$, we assign a type $\gamma$ to $\mathrm{p} 1$, so that the current constraint set includes $\varsigma \leq \gamma$, where $\varsigma=\beta \leftarrow\left(\mathrm{x}, t_{1}\right) \leftarrow$ $\left(\mathrm{y}, t_{2}\right)$ is an instance of the return type of Point.

The meta variable $M$ represents a set of member names and it ranges over set variable $\mathcal{M}$, empty set, a singleton set $\{m\}$, the union or intersection of two sets. We use set variable $\mathcal{M}$ in the typing rule for method calls to model the implicit extension of method arguments.

$$
\begin{aligned}
M:= & \mathcal{M} \quad \text { member set variable } \\
& \mid \\
& \{m\} \\
& M \cup M^{\prime} \\
& M \cap M^{\prime}
\end{aligned}
$$

A recursively constrained type $\kappa$ has the form of $\tau \backslash C$. where $C$ consists of constraints of the form:

$$
\begin{aligned}
\tau & \leq\left[m^{\psi}: t\right], \text { where } \psi \in\{+,-\} \\
\tau & \leq \tau^{\prime}, \quad \text { where } \tau^{\prime} \neq(t, M), \tau^{\prime} \neq \text { null } \\
m & \in \tau \\
\mathcal{M} & \subseteq M
\end{aligned}
$$

In addition, if a function type $\tau^{\prime}$ appears in $\tau \leq \tau^{\prime}$, then $\tau^{\prime}$ has the form $\overline{(t, \mathcal{M})} \rightarrow t$.

\subsection{Constraint closure}

The definition of a closed set of constraints is based on the closure rules in Figure 4. The rules (C1) to (C5) check type constraints while the rules (M1) to (M4) are for member constraints. We write $\mathrm{cls}(C)$ to represent the closure of a constraint set $C$.

Rule $(\mathrm{C} 1)$ and $(\mathrm{C} 2)$ propagate type upper bound $w$ that includes base types, function types, and field types (of the form $\left[m^{\psi}: t\right]$ 
$\{\tau \leq t, t \leq w\} \subseteq C \Rightarrow \tau \leq w \in C$

$(t, M) \leq w \in C \quad \Rightarrow \quad t \leq w \in C$

$$
\overline{(t, M)} \rightarrow \tau \leq \overline{\left(t^{\prime}, \mathcal{M}\right)} \rightarrow t \in C \quad \Rightarrow \quad\left\{\overline{t^{\prime} \leq t}, \overline{\mathcal{M} \subseteq M}, \tau \leq t\right\} \subseteq C
$$

$t \in$ field $_{C}(\varsigma, m),\left\{\tau \leq t, \varsigma \leq\left[m^{+}: t^{\prime}\right]\right\} \subseteq C \Rightarrow \tau \leq t^{\prime} \in C$ $\varsigma \leq t \in C, \quad \varsigma$ derive $_{C} \varsigma^{\prime} \Rightarrow \varsigma^{\prime} \leq t \in C$

$\left\{m \in \tau, \tau^{\prime} \leq \tau\right\} \subseteq C \Rightarrow\left(m \in \tau^{\prime}\right) \in C$

$\{m \in(\tau, M)\} \subseteq C, m \notin \operatorname{Upper}_{C}(M) \Rightarrow(m \in \tau) \in C$

$\left\{m \in\left(\varsigma_{1}, \varsigma_{2}\right)\right\} \subseteq C \quad \Rightarrow \quad\left\{m \in \varsigma_{1}, m \in \varsigma_{2}\right\} \subseteq C$

$\left\{m \in \varsigma \leftarrow\left(m^{\prime}, t\right)\right\} \subseteq C, m \neq m^{\prime} \quad \Rightarrow \quad(m \in \varsigma) \in C$

Figure 4. Definition of a closed constraint set $C$, where meta variable $w$ ranges over num, bool, $\overline{(t, M)} \rightarrow \tau$, and $\left[m^{\psi}: t\right], \psi \in\{+,-\}$.

where $\psi \in\{+,-\})$. In rule (C3), we write $\overline{t^{\prime} \leq t}$ for $t_{i}^{\prime} \leq t_{i}$ and $\overline{\mathcal{M} \subseteq M}$ for $\mathcal{M}_{i} \subseteq M_{i}$, where $i \in\{1,2\}$.

Rule (C4) generates constraints for reading the member $m$ of a pro-type $\varsigma$. The rule uses a function field $C(\varsigma, m)$ (defined below) that returns all possible types of the member $m$ in $\varsigma$. If a pro-type $\varsigma$ has the member $m$ added indirectly through function/method calls, then $\varsigma \leq\left[m^{-}: t\right] \in C$ for some type variable $t$. The function $\mathrm{fld}_{C}(\varsigma, \bar{m})$ returns the types of member $m$ directly added to $\varsigma$.

$$
\begin{aligned}
\operatorname{field}_{C}(\varsigma, m) & =\left\{t \mid \varsigma \leq\left[m^{-}: t\right] \in C\right\} \cup \operatorname{fld}_{C}(\varsigma, m) \\
\operatorname{fld}_{C}(\varsigma \leftarrow(m, t), m) & =\{t\} \\
\text { fld }_{C}\left(\varsigma \leftarrow\left(m^{\prime}, t\right), m\right) & =\text { fld }_{C}(\varsigma, m) \text { where } m^{\prime} \neq m \\
\text { fld }_{C}((\varsigma, M), m) & =\text { fld }_{C}(\varsigma, m) \\
\text { fld }_{C}\left(\left(\varsigma, \varsigma^{\prime}\right), m\right) & =\text { fld }_{C}(\varsigma, m) \cup \text { fld }_{C}\left(\varsigma^{\prime}, m\right) \\
\text { fld }_{C}(\gamma, m) & =\text { fld }_{C}(\varsigma, m), \text { where } \varsigma \leq \gamma \in C \\
\text { fld }_{C}(\beta, m) & =\emptyset
\end{aligned}
$$

Rule (C5) propagates the variable type upper bound of a protype $\varsigma$ to all the pro-types derived from $\varsigma$. The rule uses a relation $\varsigma$ derive ${ }_{C} \varsigma^{\prime}$ defined for each $\varsigma^{\prime}$ derived from $\varsigma$ :

$$
\frac{\varsigma^{\prime}=(\varsigma, M)|\varsigma \leftarrow(m, t)|\left(\varsigma,,_{-}\right) \mid(-, \varsigma)}{\varsigma \text { derive }_{C} \varsigma^{\prime}} \frac{\varsigma \leq \gamma \in C}{\varsigma \text { derive }_{C} \gamma}
$$

Note that this $\varsigma$ derive $_{C} \varsigma^{\prime}$ relation is ambiguous since $\varsigma^{\prime}$ can be formed arbitrarily. However, for our purpose, we restrict $\varsigma^{\prime}$ to the pro-types that actually exist in the type environment.

To see why this rule is needed, consider the example below. Suppose that $y$ at line 4 has type $\gamma$, where $\left\{\beta \leftarrow\left(m, t_{1}\right) \leq\right.$ $\gamma$, num $\left.\leq t_{1}\right\} \subseteq C$, and $y_{1}$ has the type $t$, where $\gamma \leq t \in C$.

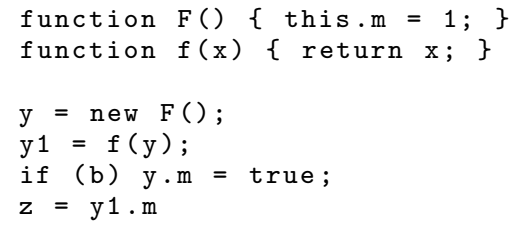

If $b$ is true, then $y$ has a new type $\gamma \leftarrow\left(m, t_{2}\right)$ after line 6, where bool $\leq t_{2} \in C$. After line 7, $z$ has the type $t^{\prime}$, where $t \leq\left[m^{+}: t^{\prime}\right] \in C$.

The variable $z$ at line 7 may be an integer or boolean. If we only have $\gamma \leq t \in C$, then Rule (C4) will only add num $\leq t^{\prime}$ to $C$ and allow $z$ be used as a number. However, since Rule (C5) adds $\gamma \leftarrow\left(m, t_{2}\right) \leq t$ to $C$, by Rule (C4), we have bool $\leq t^{\prime} \in C$, which indicates that $z$ may also be a boolean.

Rule (M1) to (M4) propagate member constraint based on subtyping relations and the structure of pro-types. If a constraint of the form $m \in \beta$ is added by the closure rules, then the constraint set is inconsistent since $\beta$ variable represents empty objects. In Rule (M2), we use a function $\operatorname{Upper}_{C}(M)$ to obtain the upper bound of $M$. Since $M$ may contain set variable $\mathcal{M}$, which may be unbounded within a particular constraint set, we let $\operatorname{Upper}_{C}^{0}(\mathcal{M})=$ $T$ for each $\mathcal{M}$, where $T$ represents the maximum set of member names. The upper bound of each $M$ is then calculated iteratively until they converge.

$$
\begin{gathered}
\operatorname{Upper}_{C}^{i+1}\left(M \cup M^{\prime}\right)=\operatorname{Upper}_{C}^{i}(M) \cup \operatorname{Upper}_{C}^{i}\left(M^{\prime}\right) \\
\operatorname{Upper}_{C}^{i+1}\left(M \cap M^{\prime}\right)=\operatorname{Upper}_{C}^{i}(M) \cap \operatorname{Upper}_{C}^{i}\left(M^{\prime}\right) \\
\operatorname{Upper}_{C}^{i}(\emptyset)=\emptyset \operatorname{Upper}_{C}^{i}(\{m\})=\{m\} \\
\operatorname{Upper}_{C}^{i+1}(\mathcal{M})=\bigcap_{\mathcal{M} \subseteq M \in C} \operatorname{Upper}_{C}^{i}(M) \\
\operatorname{Upper}_{C}(M)=\operatorname{Upper}_{C}^{i}(M) \text { if } \operatorname{Upper}_{C}^{i+1}(M)=\operatorname{Upper}_{C}^{i}(M) \forall M
\end{gathered}
$$

By Rule (M2), if a member $m$ is selected from a variable of type $(\tau, M)$ and $m$ is not in $M$, then $m$ must be defined in $\tau$, where $\tau$ may be a pro-type or obj-type.

\subsection{Constraint consistency}

We define consistency rules to verify that a closed constraints set does not contain immediate contradictions.

Constraints of the following form are immediately inconsistent:

1. $\tau \leq \tau^{\prime}$ where

(a) $\tau$ is a base type and $\tau^{\prime}$ is a different base type, or a function type or a pro-type,

(b) $\tau$ is a function type and $\tau^{\prime}$ is a base type or a pro-type,

(c) $\tau$ is a pro-type and $\tau^{\prime}$ is a base type or a function type;

2. $\tau \leq\left[m^{\psi}: t\right]$ where $\tau$ is a base type or function type;

3. null $\leq \tau$, where $\tau$ is a base type or function type;

4. $m \in \beta$.

A constraint set $C$ is consistent if no constraint in its closure $\operatorname{cls}(C)$ is immediately inconsistent.

The first two rules check constraints that are obviously incompatible. The third rule makes sure variables of base type or function type cannot be null. It is possible that variables of object types have null value. The last rule represents the error of reading undefined member. If a member $m$ is read on an object that has no definition of $m$, then the closure rule will eventually generate a constraint of the form $m \in \beta$, where $\beta$ corresponds to the initial type of the self pointer of the object's constructor. 


$$
\begin{gathered}
\frac{\forall i \in 1 . . n . \Gamma \vdash F n_{i} \quad \Gamma, \emptyset \vdash s \| \Gamma^{\prime}, C}{\Gamma \vdash F n_{i}^{i \in 1 . . n} s \| \Gamma^{\prime}, C} \\
\Gamma_{f}=\Gamma\left[\operatorname{this} \mapsto a, x \mapsto \Gamma^{\prime}, C \quad \Gamma(f)=\tau_{f} \backslash C\right. \\
\tau_{f}=\Gamma^{\prime}(a) \times \Gamma^{\prime}\left(a_{x}\right) \rightarrow \Gamma^{\prime}\left(\Gamma^{\prime}(z)\right) \\
\Gamma \vdash \text { function } f(x)\{s ; \text { return } z\} \\
\left.\Gamma_{F}=\Gamma\left[\text { this } \mapsto a, x \mapsto t_{x}, \emptyset\right)\right] \\
\tau_{F}=\Gamma^{\prime}\left(a_{x}\right) \rightarrow \Gamma_{x}, a \mapsto \beta, \Gamma_{x}(a) \\
\Gamma \vdash \text { function } F(x)\{s\}
\end{gathered}
$$

Figure 5. Typing rules for program, constructor, and function

\subsection{Type rules for functions and constructors}

The typing rules for functions and constructors are given in Figure 5 and the rules for statements are in Figure 6. The typing rules use the following notations.

A type environment $\Gamma$ is a mapping from function/constructor names to rc types and a mapping from variables to type aliases, and from type aliases to types. We use type alias to track a variable's type until the variable is assigned with a different type. A type alias $a$ is uniquely mapped to a type while several variables may be mapped to the same type alias.

For any name/variable/alias in the domain of $\Gamma$, we define

$$
\begin{aligned}
& \frac{\Gamma=[\ldots f \mapsto \kappa \ldots]}{\Gamma(f)=\kappa} \quad \frac{\Gamma=[\ldots F \mapsto \kappa \ldots]}{\Gamma(F)=\kappa} \\
& \frac{\Gamma=[\ldots y \mapsto a \ldots]}{\Gamma(y)=a} \frac{\Gamma=[\ldots a \mapsto \tau \ldots]}{\Gamma(a)=\tau}
\end{aligned}
$$

Given an initial environment $\Gamma$ with the mapping of functions/constructors to their types, a program $F n_{i}^{i \in 1 . . n} s$ is well-typed if each of the function/constructor definition $F n_{i}$ is well-typed and the main program $s$ is well-typed.

The definition of a function $f$ is well-typed given $\Gamma$ if we can construct a new environment from $\Gamma$ for the function body so that it is well-typed. In particular, the parameters are assigned extended types with empty member set such as $(t, \emptyset)$. After the function body $s$ is analyzed, the type of the parameter may be extended to $(t, M)$. We use $(t, M)$ as the type of the parameter in the function type. $M$ is used to record the members added to the parameter in $s$. Though type alias is not needed for the self pointer since it is immutable, the use of type alias $a_{x}$ in Rule (T-Fn) allows us to keep track of the extension to the parameter type $t_{x}$ in case it is assigned a different value in the function body.

Given a type environment $\Gamma$ and a constraint set $C$, the typing rules for statements derive a possibly new environment $\Gamma^{\prime}$ and constraint set $C^{\prime}$ for a statement $s$, written as $\Gamma, C \vdash s \| \Gamma^{\prime}, C^{\prime}$. Each rule implicitly enforces the consistency of the constraint set $C^{\prime}$. In Rule (T-Fn), the constraint set derived from the body of a function $f$ is the one in the rc type of $f$ defined in the initial type environment (e.g. $\Gamma(f)=\tau_{f} \backslash C$ ). This does present a problem for mutually recursive functions and we will address this in Section 5, where we also show the steps to simplify the constraint set.

\subsection{Type rules for statements}

Typing rules for statements are shown in Figure 6, where we use the following definition of $\tau \cup M$ to represent type extensions.

$$
\begin{aligned}
t \cup M & \equiv(t, M) \\
(t, M) \cup M^{\prime} & \equiv\left(t, M \cup M^{\prime}\right) \\
\varsigma \cup \mathcal{M} & \equiv(\varsigma, \mathcal{M})
\end{aligned}
$$

For an assignment $x=v$, Rule (T-Assn) finds the type of $v$ through Rule (T-Val) with the judgment $\Gamma \vdash v: \tau \backslash C$.

For each appearance of a function $f$, we can instantiate the rc type $\tau \backslash C$ of $f$ by renaming the variables (including type and set variables) in $\tau$ and $C$. For a recursively defined function, the renaming of its type within itself is the identity function.

To illustrate the typing rules, we show how the function leftMost in Figure 1 can be typed as follows:

$$
\begin{aligned}
& \Gamma \vdash \quad \text { function leftMost }\left(z_{1}, z_{2}\right)\{ \\
& x_{1}=z_{1} \cdot \operatorname{getX}() ; \\
& x_{2}=z_{2} \cdot \operatorname{getX}() ; \\
& b=x_{1} \leq x_{2} \\
& \operatorname{if}(b)\left\{r=z_{1} ;\right\} \text { else }\left\{r=z_{2} ;\right\} \\
& \text { return } r \\
&\}
\end{aligned}
$$

where $\Gamma=\{$ leftMost $\mapsto \kappa\}$ and

$$
\begin{aligned}
& \left\{t_{1} \leq\left[\operatorname{getX}^{+}: t_{3}\right], t_{2} \leq\left[\operatorname{getX} X^{+}: t_{4}\right],\right. \\
& \operatorname{getX} \in t_{1}, \operatorname{getX} \in t_{2}, \\
\kappa=t_{1} \times t_{2} \rightarrow t \backslash \quad & t_{3} \leq t_{5} \rightarrow t_{7}, t_{4} \leq t_{6} \rightarrow t_{8}, \\
& t_{1} \leq t_{3}, t_{2} \leq t_{4}, t_{7} \leq \text { num }, t_{8} \leq \text { num, } \\
& \left.t_{1} \leq t, t_{2} \leq t\right\} .
\end{aligned}
$$

By Rule (T-Fn), we construct the type environment $\Gamma_{1}$ to check the function body. so that

$$
\Gamma_{1}=\Gamma \cup\left\{z_{1} \mapsto a_{1}, a_{1} \mapsto t_{1}, z_{2} \mapsto a_{2}, a_{2} \mapsto t_{2}\right\} .
$$

Here we omit the type for self pointer since it is never used and we also omit the member set associated with parameter types since they are not extended. From this environment and an empty constraint set, we can apply statement typing rules to each statement in the function body sequentially to derive the final constraint set for the function.

For the first statement, based on Rule (T-Invk), we have

$$
\begin{aligned}
& \Gamma_{1}, \emptyset \vdash x_{1}=z_{1} \cdot \operatorname{getX}() \| \Gamma_{2}, C_{2}, \text { where } \\
& \Gamma_{2}=\Gamma_{1}\left[x_{1} \mapsto a_{1}^{\prime}, a_{1}^{\prime} \mapsto t_{7}\right] \\
& C_{2}=\left\{t_{1} \leq\left[\operatorname{getX}{ }^{+}: t_{3}\right], \operatorname{getX} \in t_{1},\right. \\
& \left.\quad t_{3} \leq t_{5} \rightarrow t_{7}, t_{1} \leq t_{5}\right\} .
\end{aligned}
$$

In $t_{5} \rightarrow t_{7}$, the type $t_{5}$ corresponds to the receiver type of the call $\mathrm{z} 1$. getX () and the parameter type is omitted since get $\mathrm{X}$ has no parameter.

Similarly, for the second statement, we have

$$
\begin{aligned}
\Gamma_{2}, C_{2} \vdash x_{2}=z_{2} \cdot \operatorname{getX}() \| \Gamma_{3}, C_{3}, \text { where } \\
\Gamma_{3}=\Gamma_{2}\left[x_{2} \mapsto a_{2}^{\prime}, a_{2}^{\prime} \mapsto t_{8}\right] \\
C_{3}=C_{2} \cup\left\{t_{2} \leq\left[\operatorname{getX} t_{4}^{+}\right], \text {getX } \in t_{2},\right. \\
\left.t_{4} \leq t_{6} \rightarrow t_{8}, t_{2} \leq t_{6}\right\} .
\end{aligned}
$$

For the third statement, we don't have a rule to check comparison expression but it can be easily added:

$$
\Gamma \vdash x \leq y \text { : bool } \backslash\{\Gamma(\Gamma(x)) \leq \text { num, } \Gamma(\Gamma(y)) \leq \text { num }\}
$$




$$
\begin{aligned}
& \Gamma \vdash n: \text { num } \backslash \emptyset \quad \Gamma \vdash b: \text { bool } \backslash \emptyset \quad \Gamma \vdash \text { null : null } \backslash \emptyset \\
& \begin{array}{r}
\hat{f}=f \text { or } \hat{f}=F \quad \Gamma(\hat{f})=\tau \backslash C \quad \Psi \text { is a renaming of the type variables in } C \\
\Gamma \vdash \hat{f}: \Psi \tau \backslash \Psi C
\end{array} \\
& \Gamma \vdash v: \tau \backslash C^{\prime} \\
& \overline{\Gamma, C \vdash x=v \| \Gamma[x \mapsto a, a \mapsto \tau], C \cup C^{\prime}} \\
& \Gamma, C \vdash x=y \| \Gamma[x \mapsto \Gamma(y)], C \\
& \frac{\Gamma\left(\Gamma\left(y^{\prime}\right)\right)=\tau^{\prime} \quad \Gamma(y)=a_{y} \quad \Gamma\left(a_{y}\right)=\tau \quad \tau \neq \varsigma \quad C^{\prime}=C \cup\left\{\tau \leq\left[m^{-}: t\right], \tau^{\prime} \leq t\right\}}{\Gamma, C \vdash y \cdot m=y^{\prime} \| \Gamma\left[a_{y} \mapsto \tau \cup\{m\}\right], C^{\prime}} \\
& \frac{\Gamma\left(\Gamma\left(y^{\prime}\right)\right)=\tau^{\prime} \quad \Gamma(y)=a_{y} \quad \Gamma\left(a_{y}\right)=\varsigma \quad \varsigma^{\prime}=\varsigma \leftarrow(m, t)}{\Gamma, C \vdash y \cdot m=y^{\prime} \| \Gamma\left[a_{y} \mapsto \varsigma^{\prime}\right], C \cup\left\{\tau^{\prime} \leq t\right\}} \\
& \frac{\Gamma \vdash F: \tau_{F} \backslash C_{F} \quad \Gamma(y)=a_{y} \quad \Gamma\left(a_{y}\right)=\tau_{y} \quad C^{\prime}=\left\{\tau_{F} \leq(t, \mathcal{M}) \rightarrow \gamma, \tau_{y} \leq t\right\}}{\Gamma, C \vdash x=\operatorname{new} F(y) \| \Gamma\left[x \mapsto a, a \mapsto \gamma, a_{y} \mapsto \tau_{y} \cup \mathcal{M}\right], C \cup C^{\prime} \cup C_{F}} \\
& \frac{\Gamma(\Gamma(y))=\tau \quad C^{\prime}=\left\{\tau \leq\left[m^{+}: t\right], m \in \tau\right\}}{\Gamma, C \vdash x=y \cdot m \| \Gamma[x \mapsto a, a \mapsto t], C \cup C^{\prime}} \\
& \Gamma(y)=a \quad \Gamma\left(y^{\prime}\right)=a^{\prime} \quad \Gamma(a)=\tau \quad \Gamma\left(a^{\prime}\right)=\tau^{\prime} \quad \Gamma^{\prime}=\Gamma\left[x \mapsto a_{x}, a_{x} \mapsto t_{x}, a \mapsto \tau \cup \mathcal{M}, a^{\prime} \mapsto \tau^{\prime} \cup \mathcal{M}^{\prime}\right] \\
& C^{\prime}=\left\{\tau \leq\left[m^{+}: t\right], t \leq\left(t_{y}, \mathcal{M}\right) \times\left(t_{y}^{\prime}, \mathcal{M}^{\prime}\right) \rightarrow t_{x}, m \in \tau, \tau \leq t_{y}, \tau^{\prime} \leq t_{y}^{\prime}\right\} \\
& \Gamma, C \vdash x=y \cdot m\left(y^{\prime}\right) \| \Gamma^{\prime}, C \cup C^{\prime} \\
& \frac{\Gamma, C \vdash s\left\|\Gamma^{\prime}, C^{\prime} \Gamma^{\prime}, C^{\prime} \vdash s^{\prime}\right\| \Gamma^{\prime \prime}, C^{\prime \prime}}{\Gamma, C \vdash s ; s^{\prime} \| \Gamma^{\prime \prime}, C^{\prime \prime}} \\
& \Gamma, C \vdash s_{1}\left\|\Gamma_{1}, C_{1} \quad \Gamma, C \vdash s_{2}\right\| \Gamma_{2}, C_{2} \quad \operatorname{mrg}\left(\Gamma_{1}, \Gamma_{2}, \Gamma^{\prime}, C^{\prime}\right) \\
& \Gamma, C \vdash \operatorname{if}(x)\left\{s_{1}\right\} \text { else }\left\{s_{2}\right\} \| \Gamma^{\prime}, C_{1} \cup C_{2} \cup C^{\prime} \cup\{\Gamma(\Gamma(x)) \leq \text { bool }\}
\end{aligned}
$$

T-Assn

T-Assn2

T-Upd

T-Upd2

T-New

T-Invk

T-Seq

T-If

Figure 6. Type rules for statements, where for each judgment $\Gamma, C \vdash s \| \Gamma^{\prime}, C^{\prime}$, it is implicit that $C^{\prime}$ is consistent.

Thus, we have

$$
\begin{aligned}
\Gamma_{3}, C_{3} & \vdash b=x_{1} \leq x_{2} \| \Gamma_{4}, C_{4}, \text { where } \\
\Gamma_{4} & =\Gamma_{3}[b \mapsto \text { bool }] \\
C_{4} & =C_{3} \cup\left\{t_{7} \leq \text { num, } t_{8} \leq \text { num }\right\} .
\end{aligned}
$$

For the fourth statement, we apply Rule (T-If) so that

$$
\Gamma_{4}, C_{4} \vdash \operatorname{if}(b)\left\{r=z_{1} ;\right\} \text { else }\left\{r=z_{2} ;\right\} \| \Gamma_{5}, C_{5} \text {, where }
$$

$$
\begin{aligned}
& \Gamma_{5}=\Gamma_{4}\left[r \mapsto a_{r}, a_{r} \mapsto t\right] \\
& C_{5}=C_{4} \cup\left\{t_{1} \leq t, t_{2} \leq t\right\} .
\end{aligned}
$$

The redundant constraint bool $\leq$ bool is omitted.

Rule (T-If) uses a predicate $\operatorname{mrg}\left(\Gamma_{t}, \Gamma_{f}, \Gamma^{\prime}, C^{\prime}\right)$ that merges the type environments of both branches $\Gamma_{t}$ and $\Gamma_{f}$ into a new environment $\Gamma^{\prime}$ and a constraint set $C^{\prime}$. By Rule (T-Assn2), the assignment in each branch of the if statement creates a new environment

$$
\begin{aligned}
\Gamma_{t} & =\Gamma_{4}\left[r \mapsto a_{t}, a_{t} \mapsto t_{1}\right], \\
\Gamma_{f} & =\Gamma_{4}\left[r \mapsto a_{f}, a_{f} \mapsto t_{2}\right] .
\end{aligned}
$$

When we merge the two environments, we get

$$
\Gamma_{4}\left[r \mapsto a_{r}, a_{r} \mapsto t\right] \text { and }\left\{t_{1} \leq t, t_{2} \leq t\right\} .
$$

Below is the definition of the merge predicates. The merging process includes the merging of mappings from variables to type aliases and the merging of mappings from type aliases to types.

$$
\begin{gathered}
\operatorname{mrg}(\Gamma, \Gamma, \Gamma, \emptyset) \quad \frac{a \notin \operatorname{dom}\left(\Gamma_{2}\right) \operatorname{mrg}\left(\Gamma_{1}, \Gamma_{2}, \Gamma, C\right)}{\operatorname{mrg}\left(\left(a \mapsto \tau, \Gamma_{1}\right), \Gamma_{2}, \Gamma, C\right)} \\
\frac{\operatorname{mrg}\left(\Gamma_{1}, \Gamma_{2}, \Gamma, C\right)}{\operatorname{mrg}\left(\Gamma_{2}, \Gamma_{1}, \Gamma, C\right)} \frac{y \notin \operatorname{dom}\left(\Gamma_{2}\right) \operatorname{mrg}\left(\Gamma_{1}, \Gamma_{2}, \Gamma, C\right)}{\operatorname{mrg}\left(\left(y \mapsto a, \Gamma_{1}\right), \Gamma_{2}, \Gamma, C\right)} \\
\frac{\varsigma_{1} \neq \varsigma_{2} \operatorname{mrg}\left(\Gamma_{1}, \Gamma_{2}, \Gamma, C\right)}{\operatorname{mrg}\left(\left(a \mapsto \varsigma_{1}, \Gamma_{1}\right),\left(a \mapsto \varsigma_{2}, \Gamma_{2}\right),\left(a \mapsto\left(\varsigma_{1}, \varsigma_{2}\right), \Gamma\right), C\right)} \\
\frac{\operatorname{mrg}\left(\Gamma_{1}, \Gamma_{2}, \Gamma, C\right)}{\operatorname{mrg}\left(\left(a \mapsto t, \Gamma_{1}\right),\left(a \mapsto(t, M), \Gamma_{2}\right),(a \mapsto t, \Gamma), C\right)} \\
\frac{\operatorname{mrg}\left(\Gamma_{1}, \Gamma_{2}, \Gamma, C\right) \tau=\left(t, M_{1} \cap M_{2}\right)}{\operatorname{mrg}\left(\left(a \mapsto\left(t, M_{1}\right), \Gamma_{1}\right),\left(a \mapsto\left(t, M_{2}\right), \Gamma_{2}\right),(a \mapsto \tau, \Gamma), C\right)} \\
\Gamma_{1}\left(a_{1}\right)=\tau_{1} \quad \Gamma_{2}\left(a_{2}\right)=\tau_{2} \quad a_{1} \neq a_{2} \\
\frac{\operatorname{mrg}\left(\Gamma_{1}, \Gamma_{2}, \Gamma, C\right) \quad C^{\prime}=C \cup\left\{\tau_{1} \leq t, \tau_{2} \leq t\right\}}{\operatorname{mrg}\left(\left(y \mapsto a_{1}, \Gamma_{1}\right),\left(y \mapsto a_{2}, \Gamma_{2}\right),(y \mapsto a, a \mapsto t, \Gamma), C^{\prime}\right)}
\end{gathered}
$$

As another example, consider the constructor Line in Figure 2 with some simplification. We can derive its type as follows: 


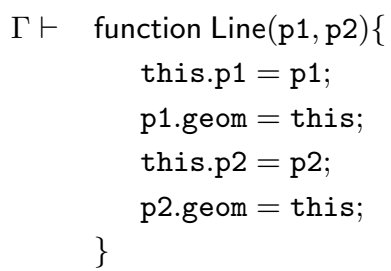

where $\Gamma=\{$ Line $\mapsto \kappa\}$,

$$
\begin{aligned}
& \left\{t_{1} \leq \text { geom }^{-}: t_{\mathrm{g} 1}\right], \\
& \left.t_{2} \leq \text { geom }^{-}: t_{\mathrm{g} 2}\right], \\
& \kappa=\left(t_{1},\{\text { geom }\}\right) \times\left(t_{2},\{\text { geom }\}\right) \rightarrow \varsigma_{2} \backslash \quad t_{1} \leq t_{\mathrm{p} 1}, t_{2} \leq t_{\mathrm{p} 2}, \\
& \left.\varsigma_{1} \leq t_{\mathrm{g} 1}, \varsigma_{2} \leq t_{\mathrm{g} 2}\right\},
\end{aligned}
$$

$\varsigma_{1}=\beta \leftarrow\left(\mathrm{p} 1, t_{\mathrm{p} 1}\right)$, and $\varsigma_{2}=\varsigma_{1} \leftarrow\left(\mathrm{p} 2, t_{\mathrm{p} 2}\right)$.

By Rule (T-Ctr), we create a type environment $\Gamma_{1}$ for the function body so that

$$
\begin{aligned}
\Gamma_{1}=\quad \Gamma[\text { this } \mapsto a, a \mapsto \beta, \\
\text { p1 } \mapsto a_{1}, a_{1} \mapsto\left(t_{1}, \emptyset\right), \\
\text { p2 } \left.\mapsto a_{2}, a_{2} \mapsto\left(t_{2}, \emptyset\right)\right]
\end{aligned}
$$

Note the typing rule assumed one parameter but the same design applies to multiple parameters.

For the first statement, we apply Rule (T-Upd2) so that

$$
\begin{aligned}
& \Gamma_{2}=\Gamma_{1}\left[a \mapsto \varsigma_{1}\right], \\
& C_{2}=\left\{\left(t_{1}, \emptyset\right) \leq t_{\mathrm{p} 1}\right\} .
\end{aligned}
$$$$
\Gamma_{1}, \emptyset \vdash \text { this.p1 }=\text { p1 } \| \Gamma_{2}, C_{2} \text { where }
$$

For simplicity, we replace $\left(t_{1}, \emptyset\right) \leq t_{\mathrm{p} 1}$ with $t_{1} \leq t_{\mathrm{p} 1}$ so that $C_{2}=\left\{t_{1} \leq t_{\mathrm{p} 1}\right\}$ since they have the same effect.

For the second statement, we apply Rule (T-Upd) so that

$$
\begin{aligned}
& \Gamma_{2}, C_{2} \vdash \text { p1.geom }=\text { this } \| \Gamma_{3}, C_{3} \text { where } \\
& \left.\Gamma_{3}=\Gamma_{2}\left[a \mapsto\left(t_{1}, \text { \{geom }\right\}\right)\right] \\
& \left.C_{3}=C_{2} \cup\left\{\left(t_{1}, \emptyset\right) \leq \text { geom }^{-}: t_{\mathrm{g} 1}\right], \varsigma_{1} \leq t_{\mathrm{g} 1}\right\} .
\end{aligned}
$$

Again, we replace $\left(t_{1}, \emptyset\right) \leq$ [geom $\left.^{-}: t_{\mathrm{g} 1}\right]$ with $t_{1} \leq$ [geom $\left.^{-}: t_{\mathrm{g} 1}\right]$ for simplicity.

The last two statements are typed the same way.

To see how Rule (T-New) is applied, consider the statement line $=$ new Line $(\mathrm{p} 1, \mathrm{p} 2)$ (line 17 of Figure 2$)$, where we assume $\mathrm{p} 1$ and $\mathrm{p} 2$ are point objects of the type $\varsigma_{\mathrm{p} 1}$ and $\varsigma_{\mathrm{p} 2}$ respectively. By Rule (T-New),

$$
\begin{aligned}
& \Gamma, C \vdash \text { line }=\text { new Line }(\mathrm{p} 1, \mathrm{p} 2) \| \Gamma^{\prime}, C^{\prime} \text { where } \\
& \Gamma^{\prime}=\Gamma[\text { line } \mapsto a, a \mapsto \gamma, \\
& \left.\quad \mathrm{p} 1 \mapsto\left(\varsigma_{\mathrm{p} 1}, \mathcal{M}_{1}\right), \mathrm{p} 2 \mapsto\left(\varsigma_{\mathrm{p} 2}, \mathcal{M}_{2}\right)\right], \\
& C^{\prime}=C \cup C_{\text {Line }} \cup \\
& \quad\left\{\tau_{\text {Line }} \leq\left(t_{1}, \mathcal{M}_{1}\right) \times\left(t_{2}, \mathcal{M}_{2}\right) \rightarrow \gamma, \varsigma_{\mathrm{p} 1} \leq t_{1}, \varsigma_{\mathrm{p} 2} \leq t_{2}\right\},
\end{aligned}
$$

and $\tau_{\text {Line }} \backslash C_{\text {Line }}$ is an instance of the rc type of the Line constructor from the judgment $\Gamma \vdash$ Line $: \tau_{\text {Line }} \backslash C_{\text {Line }}$.

If we consider the closure of $C^{\prime}$, then it contains the following constraints $C_{\mathrm{p} 1}$ related to the type of $\mathrm{p} 1$ in $\Gamma^{\prime}$ :

$\left\{\varsigma_{\mathrm{p} 1} \leq t_{1}, t_{1} \leq t_{1}^{\prime}, t_{1}^{\prime} \leq\right.$ geom $\left.^{-}: t_{\mathrm{g} 1}^{\prime}\right], \varsigma_{1}^{\prime} \leq t_{\mathrm{g} 1}^{\prime}, \mathcal{M}_{1} \subseteq\{$ geom $\left.\}\right\}$ The primed variables are unique instances of the variables in the rc type $\kappa$ of the Line function through renaming.

The typing of the statement $g=p 1$.geom (line 20 of Figure 2) generates the environment

$$
\Gamma^{\prime \prime}=\Gamma^{\prime}[\mathrm{g} \mapsto t]
$$

and the constraints

$$
C^{\prime \prime}=C^{\prime} \cup\left\{\left(\varsigma_{\mathrm{p} 1}, \mathcal{M}_{1}\right) \leq\left[\text { geom }^{+}: t\right] \text {, geom } \in\left(\varsigma_{\mathrm{p} 1}, \mathcal{M}_{1}\right)\right\} .
$$

We can see that $C^{\prime \prime}$ is consistent since $\operatorname{Upper}_{C^{\prime \prime}}\left(\mathcal{M}_{1}\right)=\{$ geom $\}$ and the closure rule (M2) does not reduce the constraint geom $\in$ $\left(\varsigma_{\mathrm{p} 1}, \mathcal{M}_{1}\right)$ to geom $\in \varsigma_{\mathrm{p} 1}$. Also, by closure rule $(\mathrm{C} 4)$, the closure of $C^{\prime \prime}$ has the constraint $\varsigma_{1}^{\prime} \leq t$. Note that $\varsigma_{1}^{\prime}$ is a pro-type of the line object and the variable $g$ is an alias of line. By closure rule (C5), the closure of $C^{\prime \prime}$ also contains $\varsigma_{2}^{\prime} \leq t$ so that the type of the variable $g$ reflects the latest state of the line object.

In summary, our typing rules are flexible enough to handle the polymorphic types and object extensions. In the appendix, we give an operational semantics for our language and show that if a program is typable then it will not access undefined object members.

\section{Type Inference}

The typing rules for statements are sufficient for type inference though the typing rules for functions and constructors do not consider mutually recursive definitions. Also, we need to simplify the type constraint set for each inferred rc type to reduce its size.

Each time a function is used, we instantiate its rc type by Rule (T-Val), which introduces fresh variables to the constraint set, but this can cause the constraint sets inferred from mutually recursive functions to have unbounded size. To solve this problem, we adopt a simple strategy that mutually recursive functions have monomorphic types when they are used among themselves, while they have polymorphic types elsewhere. When we apply Rule (TVal) for monomorphic function types, we can let the renaming $\Psi$ be identity function. While this may be restrictive for recursively defined functions, as a future work, we hope to find evidence that this is acceptable for practical programs.

$$
\begin{aligned}
& \Gamma_{1}=\emptyset \\
& \vdash_{\text {inf }} F n_{i}^{i \in \cup_{j \in 1 . . k-1} S_{j}} \| \Gamma_{k} \text { if } k>1 \\
& \frac{\Gamma_{k} \vdash_{\text {inf }} F n_{i}^{i \in S_{k}} \| \Gamma_{k+1}}{\vdash_{\text {inf }} F n_{i}^{i \in \bigcup_{j \in 1 . . k} S_{j}} \| \Gamma_{k+1}} \\
& \forall i \in S_{k} . \hat{f}_{i} \text { is the name of } F n_{i} \\
& \Gamma_{k}\left[\hat{f}_{i} \mapsto t_{i}^{i \in S_{k}}\right] \vdash_{\text {inf }} F n_{i}: \tau_{i} \backslash C_{i} \\
& C=\bigcup_{i} C_{i} \quad C^{\prime}=C\left[\tau_{i} / t_{i}^{i \in S_{k}}\right] \quad C^{\prime} \text { is consistent } \\
& \frac{\Gamma_{k+1}=\Gamma_{k}\left[\hat{f}_{i} \mapsto \tau_{i} \backslash \operatorname{reach}_{C^{\prime}}^{+}\left(\tau_{i}\right)^{i \in S_{k}}\right]}{\Gamma_{k} \vdash_{\text {inf }} F n_{i}^{i \in S_{k}} \| \Gamma_{k+1}} \quad \text { I-Prog2 } \\
& \Gamma, \emptyset \vdash s \| \Gamma^{\prime}, C \\
& \Gamma=\Gamma_{f}\left[\text { this } \mapsto a, x \mapsto a_{x},\right. \\
& \left.a \mapsto(t, \emptyset), a_{x} \mapsto\left(t_{x}, \emptyset\right)\right] \\
& \frac{\tau_{f}=\Gamma^{\prime}(a) \times \Gamma^{\prime}\left(a_{x}\right) \rightarrow \Gamma^{\prime}\left(\Gamma^{\prime}(z)\right)}{\Gamma_{f} \vdash_{\text {inf }} \text { function } f(x)\{s ; \text { return } z\}: \tau_{f} \backslash C} \\
& \Gamma, \emptyset \vdash s \| \Gamma^{\prime}, C \\
& \Gamma=\Gamma_{F}\left[\text { this } \mapsto a, x \mapsto a_{x}, a \mapsto \beta, a_{x} \mapsto(t, \emptyset)\right] \\
& \tau_{F}=\Gamma^{\prime}\left(a_{x}\right) \rightarrow \Gamma^{\prime}(a) \\
& \Gamma_{F} \vdash_{\text {inf }} \text { function } F(x)\{s\}: \tau_{F} \backslash C
\end{aligned}
$$

Figure 7. Inference rules for functions

The inference rules for functions and constructors are shown in Figure 7. Informally, the type inference algorithm has three steps: 
1. Given a program $F n_{i}^{i \in 1 . . n} ; s$, create a dependency graph $(V, E)$ where $V=\{i \mid i \in 1 . . n\}$ and $(i, j) \in E$ if $F n_{i}$ depends on $F n_{j}$. Find the strongly connected components $S_{k}$, $k \in 1 . . K$ of the graph so that if $S_{j}$ depends on $S_{i}$ then $j>i$.

2. Let $\Gamma_{1}$ be empty set. For $k>1$, assume we have inferred types for each definition in $S_{j}$ where $j=1 . . k-1$ to have $\Gamma_{k}$.

For each $S_{k}$, apply inference rules to each function/constructor $F n_{i}$ where $i \in S_{k}$ so that $\Gamma_{k} \vdash_{\text {inf }} F n_{i}: \tau_{i} \backslash C_{i}$ (Rule (IProg2) in Figure 7), where $\Gamma_{i}$ maps the function/constructor in $S_{j}, j \in 1 . . k-1$ to inferred types and maps each $F n_{i}$ in $S_{k}$ to an unconstrained type variable $t_{i}$. The type inference rules for statements are the same as typing rules for statements with the following additions to extract the variable type of function/constructor in $S_{k}$.

$$
\frac{\Gamma(f)=t}{\Gamma \vdash f: t} \frac{\Gamma(F)=t}{\Gamma \vdash F: t}
$$

After obtaining the type constraints $C_{i}$, we check the consistency of $C=\bigcup_{i \in S_{k}} C_{i}$ and simplify the type constraints for each function/constructor so that $F n_{i}$ has the type of $\tau_{i} \backslash$ reach $_{C^{\prime}}^{+}\left(\tau_{i}\right)$, where $C^{\prime}$ is $C$ with each type variable $t_{i}$ of function/constructor in $S_{k}$ replaced by its inferred type $\tau_{i}$.

The definition of reach ${ }_{C}^{+}(\tau)$ is shown in Figure 8 .

3. After the types of all functions and constructors are inferred, we obtain a type environment $\Gamma_{K}$ and then check the main program $s$ with $\Gamma_{K}, \emptyset \vdash s \| \Gamma, C$, where $C$ is consistent.

Each time a function of the type $\tau_{f} \backslash C_{f}$ is used in a context with the constraint set $C$, we add $C_{f}$ to $C$. However, not all constraints in $C_{f}$ is needed since we are only interested in those that are related to the type $\tau_{f}$. Specifically, $\tau_{f}$ may only appear in a constraint of the form $\tau_{f} \leq \tau$ in $C$ (Rule (T-Upd), (T-New), and (T-Invk)). Thus, we define reach $C_{f}^{+}\left(\tau_{f}\right)$ to select the constraints in $C_{f}$ that are relevant to the closure of $C$ related to $\tau_{f} \leq \tau$.

Informally, reach $_{C}^{+}(\tau)$ finds type constraints related to the lower bounds of $\tau$ in $C$, while reach $_{C}^{-}(\tau)$ selects constraints related to the upper bounds. Redundant constraints such as $\varsigma \leftarrow(m, t) \leq$ $\left[m^{+}: t^{\prime}\right]$ are not included since they can be reduced to more basic constraints after closure operations. reach $_{C}^{+}(\varsigma)$ keeps constraints in $C$ related to $\varsigma$ itself. reach $_{C}^{+}(\mathcal{M})$ includes constraints of the form $m \in(\tau, M)$ in $C$ where $\mathcal{M}$ affects the upper bound of $M$. reach $_{C}^{-}(M)$ has constraints in $C$ related to the upper bound of $M$.

The simplification of the rc types does not affect the functions within a strong connected component since they have monomorphic types among themselves and their constraints are pooled together to check consistency (Rule (I-Prog2)). When the functions are used in other context, we need to make sure the simplification does not remove necessary constraints. That is, we want to show that if

$$
\Gamma\left[\ldots f \mapsto \tau_{f} \backslash \operatorname{reach}_{C_{f}}^{+}\left(\tau_{f}\right) \ldots\right], C \vdash s \| \Gamma^{\prime}, C^{\prime},
$$

then

$$
\Gamma\left[\ldots f \mapsto \tau_{f} \backslash C_{f} \ldots\right], C \vdash s \| \Gamma^{\prime \prime}, C^{\prime \prime},
$$

where $C^{\prime}$ and $C^{\prime \prime}$ are consistent. Since each time $f$ is used, its type is instantiated by a renaming $\Psi$, the resulting constraint set $\Psi C_{f}$ has no overlap with $C$. When we add $\Psi C_{f}$ to $C$, they have no interaction until a constraint of the form $\Psi \tau_{f} \leq \tau$ is added by the statement typing rules. We need to verify that if

$$
\Psi \text { reach }_{C_{f}}^{+}\left(\tau_{f}\right) \cup\left\{\Psi \tau_{f} \leq \tau\right\} \cup C
$$

is consistent, then so is

$$
\Psi C_{f} \cup\left\{\Psi \tau_{f} \leq \tau\right\} \cup C .
$$

Since $\Psi$ only changes the names of variables, $\Psi$ reach $C_{f}^{+}\left(\tau_{f}\right)=$ $\operatorname{reach}_{\Psi C_{f}}^{+}\left(\Psi \tau_{f}\right)$. Thus, we need the following lemma.

Lemma 5.1. Assume $C_{f}$ and $C$ are consistent and they do not have variables in common. Also, the variables in $\tau$ do not appear in $C_{f}$. If $C \cup\left\{\tau_{f} \leq \tau\right\} \cup$ reach $_{C_{f}}^{+}\left(\tau_{f}\right)$ is consistent, then $C \cup\left\{\tau_{f} \leq\right.$ $\tau\} \cup C_{f}$ is also consistent.

Let $C^{\prime}=C \cup\left\{\tau_{f} \leq \tau\right\} \cup C_{f}$ and $C^{\prime \prime}=C \cup\left\{\tau_{f} \leq\right.$ $\tau\} \cup$ reach $_{C_{f}}^{+}\left(\tau_{f}\right)$. The proof is to show that $\operatorname{cls}\left(C^{\prime}\right) \subseteq \operatorname{cls}\left(C^{\prime \prime}\right) \bar{\cup}$ $\operatorname{cls}\left(C_{f}\right)$.

Since simplification preserves the typability of functions, we can conclude that a program is typable if we can infer its types.

Theorem 5.2. If $\vdash_{\text {inf }} F n_{i}{ }^{i \in 1 . . n} \| \Gamma_{K}$ and $\Gamma_{K}, \emptyset \vdash s \| \Gamma_{K}^{\prime}, C$, then $\exists \Gamma$ such that $\Gamma \vdash F n_{i}{ }^{i \in 1 . . n}$ and $\Gamma, \emptyset \vdash s \| \Gamma^{\prime}, C^{\prime}$.

\section{Related work}

Type inference and analysis for scripting languages Anderson et al. [4] developed a type inference system for a small subset of JavaScript that supports explicit member extensions on objects and their type system ensures that the new members may only be accessed after the extensions. Their algorithm only infers monomorphic types with explicit member extension while we allow parametric polymorphism, implicit object extensions, and strong updates and unrestricted extensions to new objects.

Recency types of Heidegger and Thiemann [12] have the similar goal of preventing the access of undefined members through type-based analysis. Their approach uses two kinds of object types: singleton type and summary type, where each singleton type is associated with an abstract location and it has to be demoted to a summary type when the next object is allocated at the same location. Objects of singleton types can receive strong updates and extensions while objects of summary types are fixed. In comparison to our work, recency type system allows singleton types for object members and function parameters. However, it does not support parametric polymorphism and singleton type objects can no longer be extended after they are assigned to variables of summary types. Pro-type objects can still be extended even if they have aliases of obj-types. Also, in their formalism, abstract locations are assigned to new expressions that return empty objects. This may be restrictive for modeling constructor functions since only the most recent object created from the constructor can have singleton type.

We have previously developed a type inference algorithm for a subset of JavaScript with implicit object extensions [25]. That algorithm also uses two kinds of object types: singleton type that allows strong update and arbitrary extensions and obj-type with limited extensions. The main difference is that it does not support parametric polymorphism.

Jensen et al. [13] have implemented a practical analyzer to detect possible runtime errors of JavaScript program. Their approach is based on abstract interpretation and uses recency information. The analyzer can report the absence of errors based on some inputs but it does not infer types. Earlier work of Thiemann [22] proposed a type system for a subset of JavaScript language to detect conversion errors of JavaScript values. The type system models automatic conversions in JavaScript but it does not model recursive or flow sensitive types.

Guha et al. [11] developed a type-checker for JavaScript that combines typing and flow analysis to support the use of local control and state to reason informally about types. Their system uses tags to identify primitives, functions, and locations. They use flow analysis to insert appropriate tag checks at each program point and then apply flow-insensitive type-checker to verify the correctness. They do not support objects or recursive types. 


$$
\begin{aligned}
& \operatorname{reach}_{C}^{-}(t)=\bigcup_{t \leq w \in \operatorname{cls}(C)} \operatorname{reach}_{C}^{-}(w) \cup\{t \leq w\} \cup \bigcup_{(m \in t) \in \operatorname{cls}(C)}\{m \in t\} \\
& \operatorname{reach}_{C}^{+}(t)=\bigcup_{\tau \leq t \in \operatorname{cls}(C)} \operatorname{reach}_{C}^{+}(\tau) \cup\{\tau \leq t\} \\
& \operatorname{reach}_{C}^{-}\left(\left[m^{+}: t\right]\right)=\operatorname{reach}_{C}^{-}(t) \quad \operatorname{reach}_{C}^{-}\left(\left[m^{-}: t\right]\right)=\operatorname{reach}_{C}^{+}(t) \\
& \operatorname{reach}_{C}^{-}(\overline{(t, \mathcal{M})} \rightarrow \tau)=\operatorname{reach}_{C}^{-}(\tau) \cup \bigcup\left(\operatorname{reach}_{C}^{+}\left(t_{i}\right) \cup \operatorname{reach}_{C}^{+}\left(\mathcal{M}_{i}\right)\right) \\
& \operatorname{reach}_{C}^{+}(\overline{(t, M)} \rightarrow \tau)=\operatorname{reach}_{C}^{+}(\tau) \cup \bigcup_{i}^{i}\left(\operatorname{reach}_{C}^{-}\left(t_{i}\right) \cup \operatorname{reach}_{C}^{-}\left(M_{i}\right)\right) \\
& \operatorname{reach}_{C}^{-}(\text {num })=\operatorname{reach}_{C}^{-}(\text {bool })=\operatorname{reach}_{C}^{-}(\text {null })=\emptyset \\
& \operatorname{reach}_{C}^{+}(\text {num })=\operatorname{reach}_{C}^{+}(\text {bool })=\operatorname{reach}_{C}^{+}(\text {null })=\emptyset \\
& \operatorname{reach}_{C}^{+}((t, M))=\operatorname{reach}_{C}^{+}(t) \\
& \operatorname{reach}_{C}^{+}(\varsigma)=\operatorname{reach}_{C}(\varsigma) \cup \bigcup_{\varsigma \leq\left[m^{-}: t\right] \in \operatorname{cls}(C)}\left(\operatorname{reach}_{C}^{+}(t) \cup\left\{\varsigma \leq\left[m^{-}: t\right]\right\}\right) \\
& \operatorname{reach}_{C}((\varsigma, M))=\operatorname{reach}_{C}(\varsigma) \\
& \operatorname{reach}_{C}\left(\left(\varsigma_{1}, \varsigma_{2}\right)\right)=\operatorname{reach}_{C}\left(\varsigma_{1}\right) \cup \operatorname{reach}_{C}\left(\varsigma_{2}\right) \\
& \operatorname{reach}_{C}(\varsigma \leftarrow(m, t))=\operatorname{reach}_{C}(\varsigma) \cup \operatorname{reach}_{C}^{+}(t) \\
& \operatorname{reach}_{C}(\gamma)=\operatorname{reach}_{C}(\varsigma), \varsigma \leq \gamma \in \operatorname{cls}(C) \quad \operatorname{reach}_{C}(\beta)=\emptyset \\
& \operatorname{reach}_{C}^{-}(M)=\bigcup\left\{\mathcal{M} \subseteq M^{\prime}\right\} \\
& \operatorname{reach}_{C}^{+}(\mathcal{M})=\bigcup_{\mathcal{M} \in R_{C}(M),(m \in(\tau, M)) \in \operatorname{cls}(C)}^{\mathcal{M} \in R_{C}(M), \mathcal{M} \subseteq M^{\prime} \in \operatorname{cls}(C)}\{m \in(\tau, M)\} \\
& R_{C}(\{m\})=R_{C}(\emptyset)=\emptyset \\
& R_{C}\left(M \cup M^{\prime}\right)=R_{C}\left(M \cap M^{\prime}\right)=R_{C}(M) \cup R_{C}\left(M^{\prime}\right) \\
& R_{C}(\mathcal{M})=\{\mathcal{M}\} \cup \bigcup_{\mathcal{M} \subseteq M \in \mathrm{cls}(C)} R_{C}(M)
\end{aligned}
$$

Figure 8. Constraint simplification

Typed Scheme [23] is an explicitly typed extension of Scheme language. The extension introduced a notion of occurrence typing to account for type tests and type predicates so that the control flow information of the program can be used to assign subtypes of a parameter to distinct occurrences.

DRuby $[9,10]$ is a tool to infer types for Ruby, which is a class-based scripting language. DRuby includes a type system with features such as union, intersection types, object types, self-type, parametric polymorphism, and tuple types. Their type inference is also a constraint-based analysis.

Type inference for class and object-based languages There are a number of studies on type inference for class-based languages. Palsberg et al. [14] have developed a type inference algorithm based on ideas of flow analysis for object-oriented programs with inheritance, assignments, and late binding. The purpose is to guarantee all messages are understood while allowing polymorphic methods. The algorithm handles late binding with conditional constraints and solves the constraints by least fixed-point derivation. Similar algorithm was applied to object-based language SELF [3] that features objects with dynamic inheritance. Plevyak and Chien [18] extended this flow-based approach for better precision via an incremental algorithm. Further enhancement on precision and efficiency were made by Agesen in his Cartesian Product Algorithm [2], which was applied to type inference for Python programs to improve compiled code [20].
The rc type used in this work is adopted from the design of Eifrig et al. [8], who developed a polymorphic, constraint-based type inference algorithm for a class-based language with polymorphic types. Their goal was to mitigate the trade-off between inheritance and subtyping. The rc types are also used in a type inference algorithm for Java [24] to verify the correctness of downcasts. Their inference algorithm extends Agesen's Cartesian Product Algorithm with the ability to analyze data polymorphic programs. In comparison, their algorithms are for class-based languages while this work applies to object-based languages with dynamic extensions.

As for type inference for object-based languages, Palsberg developed efficient type inference algorithms [15] with recursive types and subtyping for Abadi Cardelli object calculus [1], which has method override and subsumption but not object extension. Similar algorithms were developed for inferring object types for an object calculus with covariant read-only fields [17] and supporting record concatenation [16].

Type inference for dynamically typed languages is not scalable to very large programs. Spoon and Shivers [21] have developed a type inference algorithm that trades precision for speed using a demand-driven approach, which solves user provided goals by possibly generating more subgoals. They manage the number of active goals with a subgoal pruning technique, which is to provide a trivially correct answer to a goal to avoid having further subgoals. The balance between precision and scalability may be achieved by choosing pruning thresholds. 
Type systems for languages with object extensions Also related is the work of Gianantonio et al. [7] on lambda calculus of objects with self-inflicted extension. They separate the members of an object type into two parts: interface part and reservation part. After an extension, the extended member moves from reservation part to the interface part. They also distinguish two kinds of object types: pro-type and obj-type. A pro-type's reservation part may be extended but no subtyping is allowed on pro-types. A pro-type may be promoted to obj-type which allows covariant subtyping but objtypes' reservation parts may not be extended. Our pro-type objects are similar to theirs except that our pro-type objects do not lose the ability of having strong updates even after it is assigned to parameters or fields of obj-type.

Bono and Fisher [5] proposed an imperative, first-order calculus with object extensions, which also distinguishes extensible protypes without subtyping from sealed obj-types that allow width and depth subtyping. Their objective is to show that Java-style classes and mixins can be encoded in their calculus through object extensions and encapsulation.

\section{Conclusion}

We have presented a polymorphic constraint-based type inference algorithm for a small subset of JavaScript. The goal is to prevent accessing an object's member before it is defined. The type system supports explicit and implicit extension to objects and allow strong updates to new objects. Our type inference algorithm is modular so that large programs can be checked incrementally. The inferred rc-types can be simplified so that their constraint sets do not grow with the size of the program. We have implemented a prototype for checking simple JavaScript programs based on the presented algorithm and the source code is available at http://guangzhou.cs. uwm.edu/javascript.

Our primary focus is to keep track of member addition/update to objects during and after object initialization, which can be useful for some programs that exhibit this behavior [19]. However, our system is lack of many important features found in real world JavaScript programs such as prototypes, variadic functions, eval function, member deletion, runtime type tests, and objects as associative arrays. For future work, we would like to investigate the addition of prototypes and runtime type tests.

\section{References}

[1] M. Abadi and L. Cardelli. A Theory of Objects. Springer-Verlag New York, Inc., Secaucus, NJ, USA, 1996.

[2] O. Agesen. The Cartesian Product Algorithm: Simple and Precise Type Inference Of Parametric Polymorphism. In Proceedings of the 9th European Conference on Object-Oriented Programming (ECOOP'95), pages 2-26, 1995.

[3] O. Agesen, J. Palsberg, and M. I. Schwartzbach. Type Inference of SELF. In Proceedings of the 7th European Conference on ObjectOriented Programming (ECOOP'93), pages 247-267, 1993.

[4] C. Anderson, S. Drossopoulou, and P. Giannini. Towards Type Inference for JavaScript. In 19th European Conference on Object-Oriented Programming (ECOOP'05), pages 428-452, July 2005.

[5] V. Bono and K. Fisher. An Imperative, First-Order Calculus with Object Extension. In the 12th European Conference on Object-Oriented Programming (ECOOP'98), pages 462-497, 1998.

[6] D. Crockford. JavaScript: The Good Parts. O'Reilly Media, 2008.

[7] P. Di Gianantonio, F. Honsell, and L. Liquori. A Lambda Calculus of Objects With Self-Inflicted Extension. In Object-Oriented Programming Systems, Languages, and Applications (OOPSLA'98), pages 166-178, 1998.

[8] J. Eifrig, S. Smith, and V. Trifonov. Sound Polymorphic Type Inference for Objects. SIGPLAN Not., 30(10):169-184, 1995.
[9] M. Furr, J.-h. D. An, and J. S. Foster. Profile guided static typing for dynamic scripting languages. In Object-Oriented Programming Systems, Languages, and Applications (OOPSLA'09), 2009.

[10] M. Furr, J.-h. D. An, J. S. Foster, and M. Hicks. Static type inference for Ruby. In Proceedings of the 2009 ACM symposium on Applied Computing (SAC'09), pages 1859-1866, 2009.

[11] A. Guha, C. Saftoiu, and S. Krishnamurthi. Typing local control and state using flow analysis. In Proceedings of the 20th European conference on Programming languages and systems, pages 256-275, 2011.

[12] P. Heidegger and P. Thiemann. Recency Types for Analyzing Scripting Languages. In Proceedings of the 24th European Conference on Object-Oriented Programming (ECOOP'10), pages 200-224, 2010.

[13] S. H. Jensen, A. Møller, and P. Thiemann. Type Analysis for JavaScript. In 16th International Static Analysis Symposium (SAS'09), August 2009.

[14] N. Oxhøj, J. Palsberg, and M. I. Schwartzbach. Making Type Inference Practical. In Proceedings of the European Conference on ObjectOriented Programming (ECOOP'92), pages 329-349, 1992.

[15] J. Palsberg. Efficient Inference of Object Types. Inf. Comput., 123(2): 198-209, 1995

[16] J. Palsberg and T. Zhao. Type Inference for Record Concatenation and Subtyping. Inf. Comput., 189(1):54-86, 2004.

[17] J. Palsberg, T. Zhao, and T. Jim. Automatic discovery of covariant read-only fields. ACM Trans. Program. Lang. Syst., 27(1):126-162, 2005.

[18] J. Plevyak and A. A. Chien. Precise Concrete Type Inference for Object-Oriented Languages. In Proceedings of the 9th annual conference on Object-oriented programming systems, language, and applications (OOPSLA'94), pages 324-340, 1994.

[19] G. Richards, S. Lesbrene, B. Burg, and J. Vitek. An Analysis of the Dynamic Behavior of JavaScript Programs. In Proceedings of the ACM Programming Language Design and Implementation Conference (PLDI), June 2010.

[20] M. Salib. Faster than C: Static Type Inference with Starkiller. In in PyCon Proceedings, Washington DC, pages 2-26, 2004.

[21] S. A. Spoon and O. Shivers. Demand-Driven Type Inference with Subgoal Pruning: Trading Precision for Scalability. In Proceedings of the 18th European Conference on Object-Oriented Programming, (ECOOP'04), pages 51-74, 2004.

[22] P. Thiemann. Towards a Type System for Analyzing JavaScript Programs. In 14th European Symposium on Programming (ESOP'05), pages 408-422, 2005.

[23] S. Tobin-Hochstadt and M. Felleisen. The design and implementation of typed scheme. In Proceedings of the 35th annual ACM SIGPLANSIGACT symposium on Principles of programming languages, POPL '08, pages 395-406, 2008.

[24] T. Wang and S. F. Smith. Precise Constraint-Based Type Inference for Java. In Proceedings of the 15th European Conference on ObjectOriented Programming (ECOOP'01), pages 99-117, 2001.

[25] T. Zhao. Type inference for scripting languages with implicit extension. In International Workshop on Foundations of Object-Oriented Languages, 2010. 


\section{A. Semantics and Type Soundness}

\section{A.1 Operational semantics}

We define a big-step semantics for our language in Figure 9. First, we give a few definitions used in the semantics.

A heap $H$ is a mapping from object labels $\iota$ to object values $O$, which maps member names to values. A value $v$ is either an object label, a function name, a primitive value, or null.

$$
\begin{array}{ll}
v & ::=\iota|f| n|b| \text { null } \\
o & ::=\left\{m_{i} \mapsto v_{i}{ }^{i \in 1 . . n}\right\} \\
H & ::=\left\{\iota_{i} \mapsto o_{i}{ }^{i \in 1 . . n^{\prime}}\right\}
\end{array}
$$

We can extract the object value from the heap through its label.

$$
\frac{H=\{\ldots \iota \mapsto o \ldots\}}{H(\iota)=o}
$$

Similarly, we can select a member from an object value through member name if the member is defined in the object.

$$
\frac{o=\{\ldots m \mapsto v \ldots\}}{o(m)=v}
$$

Otherwise, $o(m)=$ undef, which says $m$ is undefined in $o$. Note that undef is not the undefined property in JavaScript.

We use the symbol $\chi$ to represent a stack that maps local variables to to their values and maps a special variable $\mathrm{FT}$ to the the declarations of functions and constructors.

$$
\chi::=\left\{y_{i} \mapsto v_{i}^{i \in 1 . . n}, \mathrm{FT} \mapsto F n_{j}^{j \in 1 . . n^{\prime}}\right\}
$$

We can find the value of a name $y$ from the stack if it is in the domain of the stack.

$$
\frac{\chi=\{\ldots y \mapsto v \ldots\}}{\chi(y)=v}
$$

If $y$ is not defined in the domain of $\chi$, then $\chi(y)=$ undef. Moreover, lookup $\left(f, F n_{i}^{i \in 1 . . n}\right)=F n_{j}$ if $F n_{j}$ is the declaration of the function $f$ and $\operatorname{lookup}\left(F, F n_{i}^{i \in 1 . . n}\right)=F n_{j}$ if $F n_{j}$ is the declaration of the constructor $F$, where $j \in 1$..n.

The reduction of a statement is written in form of $H, \chi, s \leadsto$ $H^{\prime}, \chi^{\prime}$, which means that the execution of a statement $s$ given the configuration of a heap $H$ and a stack $\chi$ results in a new configuration $H^{\prime}, \chi^{\prime}$.

The reduction rules are mostly straightforward and they do not consider runtime errors, which will be defined next. A statement $s$ can write to a variable $x$ not defined in $\chi$ and after the execution of $s, \chi$ is extended with the definition of $x$. The reduction of if statement uses a predicate trim to remove variables that are not defined in both branches.

$$
\frac{\forall i=1,2 . \chi_{i}^{\prime}=\left\{y \mapsto \chi_{i}(y) \mid y \in \operatorname{dom}\left(\chi_{1}\right) \cap \operatorname{dom}\left(\chi_{2}\right)\right\}}{\operatorname{trim}\left(\chi_{1}, \chi_{2}, \chi_{1}^{\prime}, \chi_{2}^{\prime}\right)}
$$

\section{A.1.1 Runtime errors}

Since big step semantics cannot distinguish a program stuck with runtime error from divergence, we define rules to propagate runtime errors during the computation. The first type of error is due to accessing an undefined member of an object or invoking a object property that is not a function. We use a special configuration error to denote the result of the computation as shown in Figure 10. We will show that a well-typed program will not result in error. The second type of error is due to deferencing a null pointer, which is represented by a special configuration nullPtrEx as shown in Figure 11 . We tolerate this type of error.

$$
\begin{aligned}
& P=F n_{i}^{i \in 1 . . n} s \quad \chi^{\prime}=\left\{\mathrm{FT} \mapsto F n_{i}^{i \in 1 . . n}\right\} \\
& \emptyset, \chi^{\prime}, s \leadsto H, \chi \\
& \emptyset, \emptyset, P \leadsto H, \chi \\
& \operatorname{lookup}(F, \chi(\mathrm{FT}))=\text { function } F\left(x_{p}\right)\{s\} \\
& \chi^{\prime}=\left\{\text { this } \mapsto \iota, x_{p} \mapsto \chi(y), \mathrm{FT} \mapsto \chi(\mathrm{FT})\right\} \\
& \frac{\iota \notin \operatorname{dom}(H) H[\iota \mapsto\{\}], \chi^{\prime}, s \leadsto H^{\prime}, \chi^{\prime \prime}}{H, \chi, x=\operatorname{new} F(y) \sim H^{\prime}, \chi[x \mapsto \iota]} \\
& \frac{H(\chi(y))(m)=v}{H, \chi, x=y \cdot m \sim H, \chi[x \mapsto v]} \\
& H(\chi(y))(m)=f \\
& \text { lookup }(f, \chi(\mathrm{FT}))=\text { function } f\left(x_{p}\right)\left\{s ; \text { return } y^{\prime \prime} ;\right\} \\
& \chi^{\prime}=\left\{\text { this } \mapsto \chi(y), x_{p} \mapsto \chi\left(y^{\prime}\right), \mathrm{FT} \mapsto \chi(\mathrm{FT})\right\} \\
& H, \chi^{\prime}, s \leadsto H^{\prime}, \chi^{\prime \prime} \\
& H, \chi, x=y \cdot m\left(y^{\prime}\right) \leadsto H^{\prime}, \chi\left[x \mapsto \chi^{\prime \prime}\left(y^{\prime \prime}\right)\right] \\
& \frac{H(\chi(y))=o \quad H^{\prime}=H\left(\chi(y) \mapsto o\left[m \mapsto \chi\left(y^{\prime}\right)\right]\right)}{H, \chi, y \cdot m=y^{\prime} \leadsto H^{\prime}, \chi} \\
& H, \chi, x=y \leadsto H, \chi[x \mapsto \chi(y)] \\
& \frac{z=n|b| f}{H, \chi, x=z \sim H, \chi[x \mapsto z]} \\
& \frac{H, \chi, s \leadsto H^{\prime}, \chi^{\prime} \quad H^{\prime}, \chi^{\prime}, s^{\prime} \leadsto H^{\prime \prime}, \chi^{\prime \prime}}{H, \chi, s ; s^{\prime} \leadsto H^{\prime \prime}, \chi^{\prime \prime}} \\
& H, \chi, s_{1} \leadsto H_{1}, \chi_{1} \quad H, \chi, s_{2} \leadsto H_{2}, \chi_{2} \\
& (\chi(x)=\text { true } \wedge i=1) \vee(\chi(x)=\text { false } \wedge i=2) \\
& \operatorname{trim}\left(\chi_{1}, \chi_{2}, \chi_{1}^{\prime}, \chi_{2}^{\prime}\right) \\
& H, \chi, \text { if }(x)\left\{s_{1}\right\} \text { else }\left\{s_{2}\right\} \leadsto H_{i}, \chi_{i}^{\prime}
\end{aligned}
$$

Figure 9. Operational semantics where the reduction rules of statements assume an implicit function table FT that maps each function/constructor name to its declaration.

\section{A.2 Type soundness}

For type soundness proof, we define an invariant that holds in each reduction step. The invariant is written as $\Sigma, \Gamma, C \vdash H, \chi$, which means that the heap $H$ and stack $\chi$ are well-formed under the environments $\Sigma$ and $\Gamma$, and constraint set $C$, where $\Sigma$ maps object labels to their types: $\Sigma=\left\{\iota_{i} \mapsto \varsigma_{i}{ }^{i \in 1 . . n}\right\}$.

The judgment $\Sigma, \Gamma, H, C \vdash v: \tau$ denotes that the value $v$ is well-typed with the type $\tau$. Here we use a function $\operatorname{Lower}_{C}(\tau)$ to retrieve the set of lower bound types of $\tau$.

$$
\begin{gathered}
\operatorname{Lower}_{C}(t)=\bigcup_{\{\tau \leq t \in \operatorname{cls}(C)\}} \operatorname{Lower}_{C}(\tau) \\
\frac{\tau \neq t \wedge \tau \neq(t, M)}{\operatorname{Lower}_{C}(\tau)=\{\tau\}} \quad \operatorname{Lower}_{C}((t, M))=\operatorname{Lower}_{C}(t)
\end{gathered}
$$

Given a constraint set $C$, a value is well-typed with the type $\tau$ if the lower bounds of $\tau$ in $C$ includes the actual type of the value.

$\frac{\text { num } \in \operatorname{Lower}_{C}(\tau)}{\Sigma, \Gamma, H, C \vdash n: \tau} \frac{\text { bool } \in \operatorname{Lower}_{C}(\tau)}{\Sigma, \Gamma, H, C \vdash b: \tau} \frac{\text { null } \in \operatorname{Lower}_{C}(\tau)}{\Sigma, \Gamma, H, C \vdash \text { null }: \tau}$




$$
\begin{aligned}
& \frac{H(\chi(y))(m)=\text { undef }}{H, \chi, x=y . m \leadsto \text { error }} \\
& \frac{H, \chi, s \leadsto \text { error or }\left(H, \chi, s \leadsto H^{\prime}, \chi^{\prime} \wedge H^{\prime}, \chi^{\prime}, s^{\prime} \leadsto \text { error }\right)}{H, \chi, s ; s^{\prime} \leadsto \text { error }} \\
& H(\chi(y))(m)=\text { undef or } \\
& H(\chi(y))(m)=f \wedge f \notin \operatorname{dom}(\chi) \text { or } \\
& \chi(f)=\text { function } f\left(x_{p}\right)\left\{s \text {; return } y^{\prime \prime} ;\right\} \\
& \frac{\chi^{\prime}=\left\{\text { this } \mapsto \chi(y), x_{p} \mapsto \chi\left(y^{\prime}\right)\right\} \quad H, \chi^{\prime}, s \leadsto \text { error }}{H, \chi, x=y \cdot m_{j}\left(y^{\prime}\right) \leadsto \text { error }} \\
& F \notin \operatorname{dom}(\chi) \text { or } \\
& \chi(F)=\text { function } F\left(x_{p}\right)\{s\} \quad \iota \notin \operatorname{dom}(H) \quad H^{\prime}=H[\iota \mapsto\{\}] \\
& \chi^{\prime}=\left\{\text { this } \mapsto \iota, x_{p} \mapsto \chi(y)\right\} \quad H^{\prime}, \chi^{\prime}, s \leadsto \text { error } \\
& H, \chi, x=\text { new } F(y) \leadsto \text { error }
\end{aligned}
$$

Figure 10. Error of accessing undefined members or functions

$$
\begin{aligned}
& \frac{\chi(y)=\text { null }}{H, \chi, x=y \cdot m \leadsto \text { nullPtrEx }} \\
& \frac{\chi(y)=\text { null }}{H, \chi, y \cdot m=y^{\prime} \leadsto \text { nullPtrEx }} \\
& H, \chi, s \leadsto \text { nullPtrEx or } \\
& \frac{H, \chi, s \leadsto H^{\prime}, \chi^{\prime} \quad H^{\prime}, \chi^{\prime}, s^{\prime} \leadsto \text { nullPtrEx }}{H, \chi, s ; s^{\prime} \leadsto \text { nullPtrEx }} \\
& \chi(y)=\text { null or } \\
& H(\chi(y))(m)=f \quad \chi(f)=\text { function } f\left(x_{p}\right)\left\{s ; \text { return } y^{\prime \prime} ;\right\} \\
& \frac{\chi^{\prime}=\left\{\text { this } \mapsto \chi(y), x_{p} \mapsto \chi\left(y^{\prime}\right)\right\} \quad H, \chi^{\prime}, s \leadsto \text { nullPtrEx }}{H, \chi, x=y \cdot m_{j}\left(y^{\prime}\right) \leadsto \text { nullPtrEx }} \\
& \iota \notin \operatorname{dom}(H) \quad H^{\prime}=H[\iota \mapsto\{\}] \quad \chi(F)=\text { function } F\left(x_{p}\right)\{s\} \\
& \chi^{\prime}=\left\{\text { this } \mapsto \iota, x_{p} \mapsto \chi(y)\right\} \quad H^{\prime}, \chi^{\prime}, s \leadsto \text { nullPtrEx } \\
& H, \chi, x=\text { new } F(y) \leadsto \text { nullPtrEx }
\end{aligned}
$$

Figure 11. Null pointer exception

Given a constraint set $C$, a function value is well-typed with the type $\tau$, if after renaming of variables, the constraint set of the function value is included in $C$ and the renamed function type is a lower bound of $\tau$ in $C$.

$$
\begin{array}{r}
\Gamma(f)=\tau_{f} \backslash C_{f} \quad \Psi \text { is a renaming of the variables in } C_{f} \\
\Psi \tau_{f} \in \operatorname{Lower}_{C}(\tau) \quad \Psi C_{f} \subseteq C
\end{array}
$$

Recall that a constraint $m \in \tau$ represents the reading of $m$ on $\tau$ and this access is safe given a constraint set $C$ if $C \cup\{m \in \tau\}$ is consistent. We use the judgement $C \vdash m \in \tau$ to represent this.

$$
\frac{C \cup\{m \in \tau\} \text { is consistent }}{C \vdash m \in \tau}
$$

The environment $\Sigma$ is only used in the proof of type soundness and we make sure that it maps each object label $\iota$ to the most current pro-type associated with $\iota$. For a label $\iota$ to be well-typed with the type $\tau, \Sigma(\iota)$ must be a lower bound of $\tau$ in $C$. Also, for each $m$ that can be accessed through $\tau, H(\iota)(m)$ must be well-typed for any type variable $t$ with the constraint set $C \cup\left\{\tau \leq\left[m^{+}: t\right]\right\}$. This constraint $\tau \leq\left[m^{+}: t\right]$ represents a read access of $m$ on $\tau$.

$$
\begin{gathered}
\Sigma(\iota)=\varsigma \quad \varsigma \in \operatorname{Lower}_{C}(\tau) \\
\frac{\forall m . C \vdash m \in \tau \Rightarrow \Sigma, \Gamma, H, C \cup\left\{\tau \leq\left[m^{+}: t\right]\right\} \vdash H(\iota)(m): t}{\Sigma, \Gamma, H, C \vdash \iota: \tau}
\end{gathered}
$$

We define a well-formed type $\tau$ with the judgement $C \vdash \tau$. An extended type $(\tau, M)$ is well-formed if for each member $m$ in $M$, there exists a corresponding constraint $\tau \leq\left[m^{-}\right.$: _] in the closure of the current constraint set $C$. This constraint represents the writing of the member $m$ to $\tau$. Other kinds of types are wellformed by default.

$$
\frac{\forall m \in \operatorname{Upper}_{C}(M) .(\tau, M) \leq\left[m^{-}:\right] \in \operatorname{cls}(C)}{C \vdash(\tau, M)} \frac{\tau \neq(t, M)}{C \vdash \tau}
$$

Using the above definitions, we define the program invariant as:

$$
\begin{gathered}
\forall \iota . \iota \in \operatorname{dom}(\Sigma) \Leftrightarrow \iota \in \operatorname{dom}(H) \\
\forall y . y \in \operatorname{dom}(\Gamma) \Leftrightarrow y \in \operatorname{dom}(\chi) \\
\forall F n \in \chi(\mathrm{FT}) . \Gamma_{\text {init }} \vdash F n \\
\forall y \in \operatorname{dom}(\chi) . \Gamma(\Gamma(y))=\tau \quad \Sigma, \Gamma, H, C \vdash \chi(y): \tau \quad C \vdash \tau \\
\Sigma, \Gamma, C \vdash H, \chi
\end{gathered}
$$

The judgment $\Sigma, \Gamma, C \vdash H, \chi$ says that the heap $H$ and stack $\chi$ are well-formed with respect to the environments $\Sigma$ and $\Gamma$, and constraint set $C$. For this invariant to hold, the domains of $H$ and $\Sigma$ must be the same and the domains of $\chi$ and $\Gamma$ have the same set of variables. Also, each variable in $\chi$ must be well-typed and each definition in $\chi(\mathrm{FT})$ is well-typed with the initial environment $\Gamma_{\text {init }}$, which only contains type mappings for functions and constructors.

Lemma A.1 shows that the execution of a well-typed statement cannot lead to errors caused by accessing undefined object members or functions. Also, the execution of a well-typed statement will result in a well-formed heap and stack.

Lemma A.1. If $\Sigma, \Gamma, C \vdash H, \chi$ and $\Gamma, C \vdash s \| \Gamma^{\prime}, C^{\prime}$, then $H, \chi, s \Varangle \rightarrow$ error, and if $H, \chi, s \leadsto H^{\prime}, \chi^{\prime}$, then $\exists \Sigma^{\prime}$ such that $\Sigma^{\prime}, \Gamma^{\prime}, C^{\prime} \vdash H^{\prime}, \chi^{\prime}$.

The proof is by straightforward induction and is omitted.

From Lemma A.1, we can conclude that well-typed programs will not lead to errors caused by accessing undefined members and invoking object properties that are not functions.

Theorem A.2 (Type Soundness). If $\Gamma \vdash P \| \Gamma^{\prime}, C$, then $\emptyset, \emptyset, P$ 九 error and if $\emptyset, \emptyset, P \leadsto H, \chi$, then $\exists \Sigma$ such that $\Sigma, \Gamma^{\prime}, C \vdash H, \chi$. 